ROMA1-1441/2006

\title{
Warped/Composite Phenomenology Simplified
}

\author{
Roberto Contino ${ }^{1,2 *}$, Thomas Kramer ${ }^{2 \dagger}$, Minho Son ${ }^{2 \ddagger}$, Raman Sundrum ${ }^{2 \S}$ \\ 1 Dipartimento di Fisica, Università di Roma "La Sapienza" and INFN \\ P.le A. Moro 2, I-00185 Roma, Italy \\ 2 Department of Physics and Astronomy, Johns Hopkins University \\ 3400 North Charles St., Baltimore, MD 21218 USA
}

\begin{abstract}
This is the first of two papers aimed at economically capturing the collider phenomenology of warped extra dimensions with bulk Standard Model fields, where the hierarchy problem is solved non-supersymmetrically. This scenario is related via the AdS/CFT correspondence to that of partial compositeness of the Standard Model. We present a purely four-dimensional, two-sector effective field theory describing the Standard Model fields and just their first Kaluza-Klein/composite excitations. This truncation, while losing some of the explanatory power and precision of the full higherdimensional warped theory, greatly simplifies phenomenological considerations and computations. We describe the philosophy and explicit construction of our two-sector model, and also derive formulas for residual Higgs fine tuning and electroweak and flavor precision variables to help identify the most motivated parts of the parameter space. We highlight several of the most promising channels for LHC exploration. The present paper focusses on the most minimal scenario, while the companion paper addresses the even richer phenomenology of the minimal scenario of precision gauge coupling unification.
\end{abstract}

\footnotetext{
*e-mail: roberto.contino@roma1.infn.it

†e-mail: tkramer@pha.jhu.edu

$\ddagger$ e-mail: mhson@pha.jhu.edu

$\S$ e-mail: sundrum@pha.jhu.edu
} 


\section{Introduction}

The known non-supersymmetric approaches to the Higgs fine-tuning problem (the Hierarchy Problem) in the Standard Model (SM) are ultimately based on the Higgs degrees of freedom being composite at several $\mathrm{TeV}$, either made from strongly-coupled constituents or extended objects such as strings. This is a theoretically challenging arena where the standard tools of perturbative, renormalizable field theory have limited applicability. Nonetheless, it is important that experiments intelligently stand watch for new $\mathrm{TeV}$-scale physics realized in this manner.

In this regard, warped compactifications of higher-dimensional spacetime provide a particularly attractive setting, for two reasons: (i) Their geometry can resolve the Hierarchy Problem non-supersymmetrically [1], (ii) When Standard Model fields are realized in the higher-dimensional "bulk" [2], quantities of central phenomenological interest become calculable in warped effective field theory, thereby describing Kaluza-Klein (KK) excitations, flavor hierarchies [3, 4, 5], rare flavor-violating processes [4, 5, 6], electroweak precision tests [7, 8, 9, 10, 11], gauge-coupling unification [12, 13], and dark matter [14]. Furthermore, this scenario incorporates or generalizes key features of other approaches. Some of these are extra-dimensional mechanisms, such as TeV-scale gravity [15, 16], Split Fermions [17], and the Hosotani mechanism [18]. But there is also a deep equivalence or duality, AdS/CFT, between this higher-dimensional physics and strongly-coupled, purely four-dimensional physics [19, 20, 21, 22]. In this way warped compactifications connect closely to the ideas of TeV-scale strong dynamics, Technicolor [23] and Walking Technicolor [24, 25], Composite Higgs [26, 27], Top-condensation [28, 29] and Topcolor [30], and partial compositeness 31.

Calculations in warped effective field theory, while doable, are not easy, and they are certainly very difficult to automate by computer. The purpose of this paper and its companion, is to provide a simplifying truncation of the kind of warped physics (with "bulk" SM fields), or equivalent composite physics, that could solve the hierarchy problem, by describing only the SM particles and the first TeV-scale excitations carrying SM charges. 1 This truncation reproduces the experimentally accessible new physics to good approximation within purely four-dimensional effective field theory. Calculations are then straightforwardly done using Feynman diagrams. The price of this truncation is that some of the explanatory power of warped compactifications is lost. Enough is retained to show how existing experimental data and bounds are satisfied, and how to identify the least tuned and most promising regions of parameter space. Roughly, the truncation is achieved by "deconstruction" [32] of the warped theory (discretization of the warped extra dimension) [33], making it appear as a variation on Little Higgs theory [34] (for a review see [35]). This truncation is very closely related, via the AdS/CFT equivalence, to the strong interactions approximation known as "vector meson dominance", in particular as expressed in the formalism of "hidden local symmetries" [36]. By this route, our work shares some aspects of the BESS approach [37] to modelling the

\footnotetext{
${ }^{1}$ The lightest SM-neutral graviton and radion excitations of the original Randall-Sundrum model [1] are less central to the phenomenology in the present context, but still interesting to consider. We have briefly described their inclusion in Appendix E.
} 
phenomenology of $\mathrm{TeV}$-scale strong dynamics, with however a tighter connection between the new states and the resolution of the hierarchy problem. Similarly, our work also shares some features with the Technicolor "straw man" models of Refs. [38.

The model developed in this paper is the minimal one consistent with the data as well the central organizing principle of partial compositeness [31]. It is closest to the TeV-scale physics of the most viable and well-developed minimal warped models [8, 9]. The models of the forthcoming companion paper additionally incorporate a simple and striking mechanism for precision gauge-coupling unification [13], with and without a weak-scale dark matter candidate [14]. Our view is that these models reflect grand principles, worthy of the significant challenges they pose to experiments, and that they are a good point of departure for thinking about how to optimize searches. But even if warped compactifications govern $\mathrm{TeV}$ physics in Nature, it is not guaranteed that any of these models is accurate in every detail. Fortunately, the simplicity of their structure makes them easy to adapt in the face of new experimental facts.

Recently, similar deconstructed approaches to TeV-scale warped phenomenology have been taken in Refs. [39, 40]. The present paper has a similar electroweak structure to that of Ref. [40, but differs considerably in other aspects such as flavor structure.

In the next section, we will give a broad overview of the physics, suppressing technical details, concluding with an outline of the remainder of the paper.

\section{Overview}

\section{$2.1 \quad$ Two sectors}

Let us sketch the central physics and how it is captured by the two physically equivalent descriptions: four-dimensional strong dynamics, and higher-dimensional warped compactification. We begin with the strong dynamics picture, where the theory is of the form

$$
\mathcal{L}=\mathcal{L}_{\text {elementary }}+\mathcal{L}_{\text {composite }}+\mathcal{L}_{\text {mixing }}
$$

There is a sector consisting of weakly-coupled elementary particles, described by $\mathcal{L}_{\text {elementary }}$. There is a second, strongly interacting sector resulting in a host of tightly bound composite states, including the Higgs doublet, described by $\mathcal{L}_{\text {composite. }}$ The elementary sector couplings are roughly $g_{e l} \sim 1$. The intra-composite forces holding each composite together are very strong, while the residual inter-composite couplings, $g_{*}$, are assumed to be weaker, 2 but still significantly stronger than the elementary couplings, $1<g_{*} \ll 4 \pi$. Other than the Higgs boson, the composites are taken to have typical masses, $M_{*}$, of very roughly TeV scale.

\subsection{Partial compositeness}

These two sectors couple to each other via the interactions of $\mathcal{L}_{\text {mixing }}$, which results primarily in mass-mixing. Consequently, mass eigenstates are non-trivial superpositions of elementary

\footnotetext{
${ }^{2}$ This is characteristic of of gauge theories with large numbers of colors.
} 
and composite particles. 3 The lightest mass eigenstates emerging from Eq. (1) are identified with the SM fields,

$$
\left.\left|S M_{n}\right\rangle=\cos \varphi_{n} \mid \text { elementary }_{n}\right\rangle+\sin \varphi_{n}\left|\operatorname{composite}_{n}\right\rangle
$$

where the mixing angles $\varphi_{n}$ parametrize the degree of "partial compositeness". The orthogonal admixtures to Eq. (2) constitute the mass eigenstates of the TeV-scale new physics. When the mass-eigenstate particles interact, the composite components interact among themselves with strength $\sim g_{*}$, and the elementary components interact among themselves with strength $\sim g_{e l}$. This rough rule captures the essence of partially composite phenomenology, which we will further elaborate on.

\subsection{Warped picture}

The warped compactification dual picture of the above physics is that the SM and heavy excitations are interpreted as the Kaluza-Klein excitations of an extra-dimensional theory, rather than describing them as mixtures of elementary and composite degrees of freedom. In this way the warped picture more naturally works in terms of the mass eigenstates. It is a deep and at first surprising result that the two apparently very different theoretical descriptions are physically equivalent. Under favorable circumstances the warped picture can give quantitative relations among the most important masses, mixing angles and couplings needed for detailed phenomenology. By comparison, the composite picture gives a clear qualitative understanding of many issues as well, but it is very difficult to pursue phenomenology quantitatively.

\subsection{Truncation}

The truncation of this paper amounts to retaining just the minimal, lowest-lying set of composite states needed in the SM admixtures. 4. Then, $\mathcal{L}_{\text {composite }}$ is taken to be a simple effective field theory for these states, while $\mathcal{L}_{\text {mixing }}$ is taken to be the general set of compositeelementary mixing mass terms (compatible with SM gauge invariance). The mass eigenstates are then the SM states of Eq. (2) and the orthogonal heavy states,

$$
\left.\left|\operatorname{heavy}_{n}\right\rangle=-\sin \varphi_{n} \mid \text { elementary }_{n}\right\rangle+\cos \varphi_{n}\left|\operatorname{composite}_{n}\right\rangle \text {. }
$$

\footnotetext{
${ }^{3}$ The SM itself contains examples of partial compositeness, where QCD represents the composite physics, that may be more familiar to the reader. The SM Higgs vacuum expectation value (vev) and the QCD chiral condensate both break the electroweak symmetry. As a result, a superposition of QCD-composite pseudoscalars and Higgs pseudoscalars (mostly the latter) are eaten by the $W, Z$, while the orthogonal superposition constitutes the observed light pions. A pion is predominantly QCD composite, with a tiny admixture of Higgs pseudoscalar. Photon- $\rho$ mixing is another example of partial compositeness. The fermionic example of positron-proton mixing is forbidden in the SM by its accidental baryon-number symmetry, but a small mixing could take place if baryon symmetry is broken by non-SM physics such as grand unification.

4 There is an exception of one charged and one neutral additional massive vector mesons, needed to have an approximate custodial symmetry to protect the electroweak $T$ parameter [8].
} 
This leads to a simple, rough pattern of couplings between the SM and heavy states. Let us consider the coupling strength for any three mass-eigenstate particles to interact, each of which could be a SM particle or a heavy particle. Given the basic rule that three elementary components interact with strength $\sim g_{e l}$ and three composite components interact with strength $\sim g_{*}$, one has

$$
\begin{aligned}
g_{S M_{1} S M_{2} S M_{3}} & \sim g_{e l} \cos \varphi_{1} \cos \varphi_{2} \cos \varphi_{3}+g_{*} \sin \varphi_{1} \sin \varphi_{2} \sin \varphi_{3} \\
g_{S M_{1} S M_{2} \text { heavy }_{3}} & \sim-g_{e l} \cos \varphi_{1} \cos \varphi_{2} \sin \varphi_{3}+g_{*} \sin \varphi_{1} \sin \varphi_{2} \cos \varphi_{3} \\
g_{S M_{1} \text { heavy }_{2} \text { heavy }_{3}} & \sim g_{e l} \cos \varphi_{1} \sin \varphi_{2} \sin \varphi_{3}+g_{*} \sin \varphi_{1} \cos \varphi_{2} \cos \varphi_{3} \\
g_{\text {heavy }_{1} \text { heavy }_{2} \text { heavy }_{3}} & \sim-g_{e l} \sin \varphi_{1} \sin \varphi_{2} \sin \varphi_{3}+g_{*} \cos \varphi_{1} \cos \varphi_{2} \cos \varphi_{3}
\end{aligned}
$$

We now use these results to understand, again roughly, the pattern of mixing angles, $\varphi$, needed in the real world, and the mechanism that has hidden $\mathrm{TeV}$ compositeness from precision tests to date.

\subsection{Compositeness and SM masses}

The SM Higgs doublet is rather special in that it must be a full composite, with no elementary component, in order to solve the Hierarchy Problem. That is: $\sin \varphi_{\text {Higgs }}=1$, $\cos \varphi_{\text {Higgs }}=0$. From Eq. (4) it follows that other (pairs of) SM particles couple to it with strength $\sim g_{*} \sin \varphi_{1} \sin \varphi_{2}$, which determines the extent to which they feel electroweak symmetry breaking (EWSB), and the masses they acquire as a result. From this we deduce that heavier SM particles are correlated with larger mixing angles. That is, the heavier SM particles have higher partial compositeness, in the operational sense that they are more strongly coupled to the new TeV-scale physics.

In this way the deep question of the origin of the large observed hierarchies in fermion masses translates in the present scenario into the question of how to obtain large hierarchies among $\varphi_{\text {fermion. }}$. It is an attractive feature of the warped compactification scenario that there is a straightforward mechanism for generating exponential hierarchies of just this type. However, this explanatory power is lost in our truncation, and the mixing angles, and the necessary hierarchies among them, are simply taken as inputs. This is a trade we make in favor of simplifying collider phenomenology considerations.

\subsection{Precision tests}

The correlation of SM mass and compositeness helps explain why the virtual effects of the new $\mathrm{TeV}$ physics have not already been seen in low-energy precision tests of the SM, given that electroweak tests are in principle sensitive to heavy physics up to $\sim 10 \mathrm{TeV}$ and flavor-changing tests are sensitive to heavy physics up to $\sim 1000 \mathrm{TeV}$. Experimentally, the maximum sensitivity applies to the lightest SM particles, but in the present scenario the lightest particles, such as light quarks and leptons, have highly $\varphi$-suppressed couplings to the new composite physics. For flavor physics, this safety mechanism generalizes the Glashow-Iliopoulos-Maiani (GIM) mechanism of the minimal SM. In the appendices of this 
paper we work out the key electroweak precision corrections due to the composite physics as a function over the model parameter space, so that due consideration can be given to electroweak precision tests. Flavor constraints are a bit less decisive due to the freedom of parameters, but in Appendix $\mathrm{A}$ we illustrate how and to what extent the generalized GIM mechanism operates within our model.

\subsection{New physics at colliders}

Eq. (4) summarizes the challenges as well as the strategy for experimentally searching for the heavy physics. Stronger couplings, $g_{*}$, at first seem promising for production of the new physics. But consider the process of fusing light SM partons to produce a heavy resonance,

$$
\mathcal{A}\left[S M_{1}+S M_{2} \rightarrow \text { heavy }\right] \propto g_{*} \varphi_{1} \varphi_{2} \cos \varphi_{\text {heavy }}-g_{\text {el }} \sin \varphi_{\text {heavy }} .
$$

The $g_{*}$ enhancement is (more than) erased by the $\varphi_{1} \varphi_{2}$ suppression. This makes it difficult, but not impossible, to produce the heavy physics. The challenge will come from seeing these events above background. Once produced, these resonances will mostly decay to the SM states with the largest mixing angles, namely the heaviest SM particles. The efficiency for identifying heavy SM particles, in particular the top and bottom quarks will be a key determinant of our ability to find the new physics. But as we will discuss, there are also special opportunities for discovery with their own special features, and these merit further study.

The minimal non-supersymmetric scenario of partial compositeness will be challenging to discover at the Large Hadron Collider (LHC), given the regions of parameter space allowed by precision tests. (The scenario with gauge coupling unification, discussed in the companion paper to this one has more easily accessible physics.) This is one of the motivations for the present paper: to make the physics as transparent as possible, to motivate hard thinking on overcoming experimental obstacles, and to exploit special opportunities. It is particularly important to have a practical measure on the parameter space to inform searches of the most motivated regions. We discuss this next.

\subsection{Residual Higgs fine tuning}

In all known solutions to the Hierarchy Problem, there remains some tension between the mechanism for solving the Higgs fine-tuning problem and the body of direct searches and precision tests, so that in effect one is forced to live with some (more modest) residual Higgs fine tuning [41]. This fact is often dubbed the "Little Hierarchy Problem". In this paper, we are not interested in tracking the residual fine tuning as a means of passing judgement on our scenario or in order to debate whether or not it is better than others in the literature. Rather, we want to use residual fine tuning to determine which regions of our own parameter space are best motivated, that is, least tuned. We choose a simple measure of residual fine tuning for this purpose,

$$
\text { Fine tuning } \sim \frac{m_{h}^{2}}{\delta m_{h \mid \text { mixing }}^{2}} .
$$


The smaller the above ratio, the more tuned a model is. We define $\delta m_{h \mid \text { mixing }}^{2}$ to be the largest radiative correction to the physical Higgs mass squared that is sensitive to the elementary sector via $\mathcal{L}_{\text {mixing. }}$ This has to be cancelled (tuned) against the tree-level Higgs mass to give the desired $m_{h}$.

A useful and elegant feature of our truncated model (and fuller descriptions) is that the leading contributions to this tuning are insensitive to the ultraviolet (UV) cutoff, $\Lambda_{U V}$, and unambiguously calculable at one loop. Mostly, this result follows by simple power-counting. Radiative corrections to the Higgs mass squared that are sensitive to the elementary sector must proceed via some virtual fluctuation from a composite to an elementary field and back to composite. These fluctuations go through the mass-mixing interactions of $\mathcal{L}_{\text {mixing }}$, which are at most of order of the composite masses, $M_{*}$, so that

$$
\delta m_{h \mid \text { mixing }}^{2} \propto M_{*}^{2} \times \text { mixing angles },
$$

with at most logarithmic sensitivity to $\Lambda_{U V}$. In detail, even this $\log \Lambda_{U V}$ is absent for the dominant corrections in our final model. In Section 8 , the UV-finite fine tuning is simply computed as a function over parameter space.

One may wonder about radiative corrections to the Higgs mass entirely originating from the composite dynamics (that is, not going through $\mathcal{L}_{\text {mixing }}$ ). In fact, in our truncated model such contributions are even larger than $\delta m_{h \mid \text { mixing }}^{2}$. However, we do not count their effect on fine tuning because we expect they would be minimized by extra structure in a fuller description of the composite sector. The classic example is when the Higgs is realized as a composite pseudo-Goldstone boson [26], such as the ordinary pion of QCD, or the AdS/CFT dual description in extra dimensions [27, 9], but there are other mechanisms as well which deserve further exploration.

\subsection{Full $t_{R}$ compositeness}

The heaviest SM particle, the top quark, requires sizeable mixing angles, $\varphi_{t_{L}, t_{R}}$. In fact, since the electroweak gauge symmetry implies $\varphi_{t_{L}}=\varphi_{b_{L}}$, and the $b_{L}$ has already been sensitively tested, we must conclude that this angle is smaller, and, to compensate, $\varphi_{t_{R}}$ is maximal. (A possible exception is if the composite sector has an enlarged custodial symmetry, a discrete subgroup of which protects the $Z b \bar{b}$ couplings from receiving large corrections [11]. In such case $\varphi_{t_{L}}$ can be large and $\varphi_{t_{R}}$ less than maximal.) Maximal $\varphi_{t_{R}}$ not only blurs the physical distinction between elementary and composite states, it also implies that quantum loops involving a virtual $t_{R}$ result in severe Higgs fine tuning. There is a natural way out of this difficulty, namely if the $t_{R}$ is in fact a fully-fledged member of the composite sector, without any elementary component. It is then an integral part of the dynamics that produces the light Higgs composite, rather than a large outside destabilizing perturbation. This is reminiscent of top-condensate [28, 29] and Topcolor [30] models, where the Higgs is realized as a composite of strong top quark interactions. 


\subsection{The error of our ways}

This paper gives a truncated model describing the SM and the lowest-lying new excitations, with mass scale $M_{*}$, with a parametrically larger UV cutoff scale, $\Lambda_{U V}$. However, in the full descriptions of warped compactifications or composite models the next-to-lowest excitations typically have masses $M_{* *} \sim 2 M_{*}$. Standard effective field theory power-counting suggests that the truncated description thereby incurs errors of order $\left(E / M_{* *}\right)^{2}$, where $E$ is the energy of a process under consideration. Since we want to consider energies large enough to create the first excitations, $E \sim M_{*}$, we can expect errors of about $1 / 4$. This may seem at first sight rather large, but the virtual effects of the $M_{* *}$-states are confined to already small corrections to low-energy precision variables or small corrections to non-resonant $M_{*}$-scale cross-sections. These corrections are frequently smaller than experimental errors. In practice we have found that the truncated model predictions do indeed match well with those of related warped compactifications. This makes the truncated model a very useful "reconnaissance tool" for our initial experimental forays into the new physics.

It should be stressed that the truncation procedure we employ for low-lying excitations, essentially discretization or "deconstruction" of the warped extra dimension, is part of a systematically improvable procedure where one makes finer and finer discretizations. In practice this proliferates the number of sectors in the model (excitation levels) and rapidly the continuum description becomes more efficient for calculation.

This concludes our non-technical overview of the relevant physics. The rest of our paper elaborates in a more quantitative way on what has been sketched so far, introducing a minimal model that gives the simplest realization of partial compositeness consistent with present data. Sections 3 and 4 introduce the two halves of our two-sector model, the "elementary sector" and "composite sector", isolated from each other for simplicity. The status of the Higgs doublet as part of the composite sector is defined. Section 5 introduces the couplings between the two sectors in the form of mass-mixing. The Lagrangian is then mass-diagonalized for phenomenological use. Section 6 describes the impact of electroweak symmetry breaking on the masses and couplings of the new physics. Section 7 makes the case that the $t_{R}$ should be a composite on par with the Higgs doublet, and shows how to simply implement this technically. In Section 8, it is shown that the dominant radiative corrections to the Higgs mass from the elementary sector are UV-finite. These finite corrections are then used to estimate Higgs fine tuning as a function of parameter space. Section 9 uses the formulas for the new-physics contributions to flavor and electroweak precision variables computed in the Appendices $\mathrm{A}$, B] and C to sketch the best motivated regions of the parameter space. Section [10] discusses the most promising channels and strategies to discover the new TeV states at the LHC. Finally, Appendix D estimates the maximal energy to which the model is internally consistent, and shows that this is well above upcoming experimental reach. Appendix E briefly describes the inclusion within the composite sector of the SM-neutral graviton and radion excitations of the Randall-Sundrum model. 


\section{$3 \quad$ Elementary Sector}

In this section we define the first building block of our minimal two-site model of partial compositeness: the elementary sector. Its field content corresponds precisely to that of the SM, but with the notable exception of the Higgs field. Indeed, the elementary fields will ultimately constitute the dominant component of the physical SM fermions and gauge bosons. The elementary gauge fields, corresponding to adjoints of the elementary gauge group $\left[S U(3)_{c} \otimes S U(2)_{L} \otimes U(1)_{Y}\right]^{e l}$, are denoted by

$$
A_{\mu} \equiv\left\{G_{\mu}, W_{\mu}, \mathcal{B}_{\mu}\right\}
$$

The SM electroweak doublet fermions are denoted by

$$
\psi_{L} \equiv\left\{q_{L i}=\left(u_{L i}, d_{L i}\right), \ell_{L i}=\left(\nu_{L i}, e_{L i}\right)\right\}, \quad i=1,2,3,
$$

or by their more individual names such as $t_{L}, \nu_{e L}, \mu_{L}$. The SM electroweak singlet fermions are denoted by 5

$$
\tilde{\psi}_{R} \equiv\left\{u_{R i}, d_{R i}, \nu_{R i}, e_{R i}\right\} .
$$

The only renormalizable interactions in this sector are gauge interactions,

$$
\mathcal{L}_{\text {elementary }}=-\frac{1}{4} F_{\mu \nu}^{2}+\bar{\psi}_{L} i \not D \psi_{L}+\overline{\tilde{\psi}}_{R} i \not D \tilde{\psi}_{R}
$$

The associated gauge couplings, $g_{e l 1}, g_{e l 2}, g_{e l 3}$, will turn out to be approximately, but not exactly, equal to the measured SM gauge couplings, $g_{1}, g_{2}, g_{3}$. For technical reasons discussed below, it is more convenient to use the standard $\mathrm{SO}(10)$ grand unified theory (GUT) convention for the hypercharge

$$
g_{1} \equiv \sqrt{\frac{5}{3}} g_{Y}, \quad Y_{G U T} \equiv \sqrt{\frac{3}{5}} Y,
$$

where $g_{Y}$ and $Y$ denote the hypercharge coupling and hypercharge generator in the usual SM convention, and $Y_{G U T}$ is defined by Eq. (12).

The elementary sector gauge dynamics obviously makes sense in isolation to the highest scales, say the Planck scale. Non-renormalizable operators made of elementary fields, like for example flavor and CP violating four-fermion interactions, will be strongly suppressed and thus negligible. Any effect of flavor and CP violation must therefore come from the composite sector and proceed through its interactions with the elementary fields.

\section{Composite Sector}

The composite sector comprises the Higgs plus what is essentially an "excited" copy of the SM fermions and gauge bosons. The basic role of these excitations is to provide a small

\footnotetext{
${ }^{5}$ The collective symbol $\tilde{\psi}_{R}$ is deliberately redundant. Normally, right-handed SM fermions are assumed to be electroweak singlets. Here, we additionally use the tilde to denote this fact and conform with our notation for the composite sector, where both chiralities of electroweak singlets ultimately appear.
} 


\begin{tabular}{|c|c|c|c|c|c|}
\hline & $S U(3)_{c}$ & $S U(2)_{L}$ & $S U(2)_{R}$ & $U(1)_{X}$ \\
\hline \hline \multicolumn{2}{|c|}{$\rho_{\mu}$} & \multicolumn{5}{|c|}{ Gauge Fields } \\
\hline \multicolumn{2}{|c|}{$(\tilde{H}, H)$} & $\mathbf{1}$ & $\mathbf{2}$ & $\mathbf{2}$ & 0 \\
\hline \multirow{2}{*}{$\chi$} & $\mathrm{Q}$ & $\mathbf{3}$ & $\mathbf{2}$ & $\mathbf{1}$ & $\frac{1}{6} \cdot \sqrt{\frac{3}{2}}$ \\
\cline { 2 - 6 } & $\mathrm{L}$ & $\mathbf{1}$ & $\mathbf{2}$ & $\mathbf{1}$ & $\left(-\frac{1}{2}\right) \cdot \sqrt{\frac{3}{2}}$ \\
\hline \multirow{4}{*}{$\tilde{\chi}$} & $\tilde{U}$ & $\mathbf{3}$ & $\mathbf{1}$ & $\mathbf{1}$ & $\frac{2}{3} \cdot \sqrt{\frac{3}{2}}$ \\
\cline { 2 - 6 } & $\tilde{D}$ & $\mathbf{3}$ & $\mathbf{1}$ & $\mathbf{1}$ & $\left(-\frac{1}{3}\right) \cdot \sqrt{\frac{3}{2}}$ \\
\cline { 2 - 6 } & $\tilde{N}$ & $\mathbf{1}$ & $\mathbf{1}$ & $\mathbf{1}$ & 0 \\
\cline { 2 - 6 } & $\tilde{E}$ & $\mathbf{1}$ & $\mathbf{1}$ & $\mathbf{1}$ & $(-1) \cdot \sqrt{\frac{3}{2}}$ \\
\hline
\end{tabular}

Table 1: Field content and quantum numbers in the composite sector of the two-site minimal model.

composite component to the physical SM fermions and gauge bosons, which determines the extent to which the latter couple to the Higgs and eventually feel electroweak symmetry breaking. This would naively suggest, in particular, that the massive vector excitations $\rho_{\mu}$ should correspond to an adjoint of $S U(3) \otimes S U(2) \otimes U(1)$. However, in order to protect the electroweak $T$ parameter as discussed below, the composite bosons must respect a larger symmetry, minimally $[S U(3) \otimes S U(2) \otimes S U(2) \otimes U(1)]$. In a controlled effective field theory, massive vector mesons must always be realized as gauge bosons of a broken gauge group. We therefore take the $\rho_{\mu}$ as gauge bosons (hence adjoint representations) of a group $\left[S U(3)_{c} \otimes S U(2)_{L} \otimes S U(2)_{R} \otimes U(1)_{X}\right]^{c o m p}$. The SM Higgs field is assumed to transform as a real bidoublet $(\tilde{H}, H)$ under $\left[S U(2)_{L} \otimes S U(2)_{R}\right]^{c o m p}$, while the quantum numbers that we adopt for the massive fermion excitations, $\chi, \tilde{\chi}$, are those given in Table 1. 6

The composite dynamics is then summarized by

$$
\begin{aligned}
\mathcal{L}_{\text {composite }}= & -\frac{1}{4} \rho_{\mu \nu}^{2}+\frac{M_{*}^{2}}{2} \rho_{\mu}^{2}+\left|D_{\mu} H\right|^{2}-V(H) \\
& +\bar{\chi}(i \not D-m) \chi+\bar{\chi}(i \not D-\tilde{m}) \tilde{\chi}-\bar{\chi}\left(Y_{* u} \tilde{H} \tilde{\chi}^{u}+Y_{* d} H \tilde{\chi}^{d}\right)+\text { h.c. },
\end{aligned}
$$

where $\tilde{\chi}^{u} \equiv\{\tilde{U}, \tilde{N}\}, \tilde{\chi}^{d} \equiv\{\tilde{D}, \tilde{E}\}$. The $\rho_{\mu}$ mass terms clearly break the composite gauge invariance completely. Technically, one can imagine that this is due to a Higgs mechanism (distinct from the electroweak Higgs mechanism) in which the associated Goldstone bosons have been eaten by the massive $\rho$ 's (that is, we are in unitary gauge), and other related

\footnotetext{
${ }^{6}$ The factor of $\sqrt{3 / 2}$ in the $U(1)_{X}$ charges comes from the relation $Y_{G U T}=\left(T^{3 R}+\xi T_{X}\right) / \sqrt{1+\xi^{2}}$, where $\xi=\sqrt{2 / 3}$ and $T^{3 R}=0$ for all the fermions. See sec. 4.1 for more details.
} 
massive fluctuations are omitted because their masses are at the cutoff scale of our composite effective field theory description. The perturbativity of our effective description then implies an upper bound on this cutoff, which we estimate in Appendix D. It can be large enough so that unspecified physics at this scale decouples from precision constraints on the model as well as collider search considerations. Closer inspection shows that the Yukawa couplings further break the gauge invariance, and this is also taken into account in Appendix D in estimating the maximal cutoff.

The mass parameters and couplings of $\mathcal{L}_{\text {composite }}$ are chosen so that the purely bosonic subsector has an $\left[S U(3)_{c} \otimes S U(2)_{L} \otimes S U(2)_{R} \otimes U(1)_{X}\right]$ global symmetry, while the Yukawa couplings break this down to an $\left[S U(3)_{c} \otimes S U(2)_{L} \otimes U(1)\right]$ global symmetry. As mentioned above, the higher bosonic symmetry will be important in protecting the electroweak $T$ parameter. It might at first seem unnatural to have a subsector of a theory enjoy a higher symmetry than the full theory, but in the present context it is technically natural because of the following observation. The Yukawa couplings do break the $S U(2)_{R}$ symmetry, grouptheoretically by $\Delta I_{R}=1 / 2$. But $\left(S U(2)_{R}\right)$-breaking in $M_{*}^{2}$ (while preserving the total $\left[S U(3)_{c} \otimes S U(2)_{L} \otimes U(1)\right]$ symmetry) is necessarily $\Delta I_{R}=2$. Thus, quadratically divergent $\left(S U(2)_{R}\right)$-breaking radiative corrections to $M_{*}^{2}$ due to the Yukawa couplings are proportional to $Y_{*}^{4}$ and arise at three (composite) loops. With the cutoff estimates of Appendix D and composite couplings large but still in the perturbative range, $g_{*}, Y_{*} \ll 4 \pi$, this breaking is negligible for our purposes. Finally, the Lagrangian $\mathcal{L}_{\text {composite }}$ is assumed to have a parity symmetry for simplicity, which means equal Yukawa couplings for both chiralities of the massive fermions.

Having sketched the composite dynamics and symmetry realizations, we will now discuss the fields and couplings in greater detail.

\subsection{Vector mesons}

In principle, the couplings $g_{*}$ and the masses $M_{*}$ of the vector mesons could be distinct for each simple subgroup of $\left[S U(3)_{c} \otimes S U(2)_{L} \otimes S U(2)_{R} \otimes U(1)_{X}\right]^{\text {comp }}$. In the present paper we will assume, purely for convenience, that these couplings are the same for the $S U(2)_{R} \otimes$ $U(1)_{X}$ subgroup of the composite gauge group. We thereby assign the parameters $\left(g_{*}, M_{*}\right)_{1}$, $\left(g_{*}, M_{*}\right)_{2}$, and $\left(g_{*}, M_{*}\right)_{3}$ to the $S U(2)_{R} \otimes U(1)_{X}, S U(2)_{L}$, and $S U(3)_{c}$ composite gauge groups. As a further simplification in subsequent numerical estimates and calculations, we will assume that all the $M_{* i}$ are similar, and that all the composite couplings are similar and moderately large, roughly $2<g_{* i}<4$.

We subdivide the vector mesons into those that will ultimately mix with the elementary gauge bosons, $\rho_{\mu}^{*}$, and those that will not, $\tilde{\rho}_{\mu}$ :

$$
\rho_{\mu}=\left\{\rho_{\mu}^{*}, \tilde{\rho}_{\mu}\right\}
$$

The $\rho_{\mu}^{*}$ transform as an adjoint of the exact global symmetry $\left[S U(3) \otimes S U(2)_{L} \otimes U(1)_{Y}\right]$, 
while the $\tilde{\rho}_{\mu}$ form an orthogonal combination: 7

$$
\rho_{\mu}^{*}=\left\{G_{\mu}^{*}, W_{\mu}^{*}, \mathcal{B}_{\mu}^{*}\right\}, \quad \tilde{\rho}_{\mu}=\left\{\tilde{W}_{\mu}^{ \pm} \equiv \frac{\tilde{W}_{1} \mp i \tilde{W}_{2}}{\sqrt{2}}, \tilde{\mathcal{B}}_{\mu}\right\} .
$$

We associate the generators $T^{1 R}, T^{2 R}$ to the fields $\tilde{W}^{1}, \tilde{W}^{2}$ and $T_{\mathcal{B}^{*}} \equiv Y_{G U T}=\left(T^{3 R}+\right.$ $\left.\xi T_{X}\right) / \sqrt{1+\xi^{2}}, T_{\tilde{\mathcal{B}}}=\left(\xi T^{3 R}-T_{X}\right) / \sqrt{1+\xi^{2}}$ respectively to $\mathcal{B}^{*}$ and $\tilde{\mathcal{B}}$. We choose $\xi=\sqrt{2 / 3}$ to match the $\mathrm{SO}(10) \mathrm{GUT}$ normalization. In this way the generator of the exactly preserved global $U(1)$ is just the hypercharge in SO(10) GUT normalization (i.e. $Y_{G U T}=\sqrt{3 / 5} Y$ ). This choice of normalization also allows us to have symmetric expressions for all gauge bosons after mass diagonalization as seen in Section 5 .

\subsection{The Higgs field}

The SM Higgs field is assumed to be entirely a composite of the new strong dynamics at $\mathrm{TeV}$ scales. Its bi-doublet transformations under $\left[S U(2)_{L} \otimes S U(2)_{R}\right]^{\text {comp }}$ are simply written by expressing the Higgs in $2 \times 2$ matrix form,

$$
(\tilde{H}, H) \rightarrow L(\tilde{H}, H) R^{\dagger}
$$

where $H$ is the usual SM Higgs doublet field for the down-type Yukawa couplings and $\tilde{H}=i \sigma^{2} H^{*}$ for the up-type Yukawa couplings. The classical Higgs potential has the usual SM form,

$$
V(H) \equiv-\mu_{H}^{2}|H|^{2}+\lambda_{H}|H|^{4},
$$

and is $\left[S U(2)_{L} \otimes S U(2)_{R}\right]^{c o m p}$-invariant.

A critical assumption we will make is that a fuller description of the composite dynamics (neglecting other sectors such as the elementary sector) would make $V(H) \equiv 0$ natural (for example, if the Higgs were realized as a Goldstone boson) and that there may in addition be perturbing dynamics in the composite sector that yield a non-vanishing but weak potential,

$$
\mu_{H}<\mathrm{TeV}, \quad \lambda_{H} \sim 1 .
$$

That is we will use this simple classical $V(H)$ to stand for the fully renormalized effective potential due to the composite dynamics alone, and assume that the desired values of $\mu_{H}, \lambda_{H}$ require no tuning before radiative corrections from the elementary sector is taken into account. Those radiative corrections to the Higgs potential that are external to the composite sector will determine our measure of fine tuning.

\subsection{Composite fermions}

The composite fermions parallel the SM fermions closely, except for the fact that they are massive Dirac fermions, rather than Weyl fermions. There are three generations of $S U(2)_{L}$

\footnotetext{
7 Our notation for the excited hypercharge boson is chosen so as not to clash with that of the excited bottom quark discussed in Section 4.3 .
} 
doublet composite Dirac fermions, denoted by

$$
\chi \equiv\left\{Q_{i}=\left(U_{i}, D_{i}\right), L_{i}=\left(N_{i}, E_{i}\right)\right\}
$$

or by more familiar names such as $(T, B), N_{\mu}, E_{\tau}$. The $S U(2)_{L}$ singlet composite Dirac fermions are denoted by

$$
\tilde{\chi} \equiv\left\{\tilde{U}_{i}, \tilde{D}_{i}, \tilde{N}_{i}, \tilde{E}_{i}\right\}
$$

or by even more familiar names such as $\tilde{T}, \tilde{B}, \tilde{N}_{\mu}, \tilde{E}_{\tau}$. Since the composite fermions have $S U(2)_{L}$ doublets and singlets with both left- and right-handed Lorentz representations, we use the presence of a tilde to denote the singlets, while we use subscripts " $L, R$ " to denote the Lorentz representation. So, for example, " $\tilde{C}_{L}$ ", denotes the left-handed Lorentz chirality of the $S U(2)_{L}$-singlet "charmed" composite quark.

The Yukawa couplings between $S U(2)_{L}$ singlets and doublets are denoted by

$$
Y_{* u}=\left\{Y_{* U}, Y_{* N}\right\}, \quad Y_{* d}=\left\{Y_{* D}, Y_{* E}\right\} .
$$

We are assuming for simplicity that $\mathcal{L}_{\text {composite }}$ has a parity symmetry, which means equal Yukawa couplings for both chiralities of the massive fermions. Here $Y_{* u}, Y_{* d}$ are matrices in ordinary generational space, which we also take to be stronger and less hierarchical than their SM equivalents, $1<Y_{* u, d} \lesssim 3$, again a reflection of the strong dynamics creating the composites. The Dirac mass terms, $m, \tilde{m}$ are free parameters of the model. They respect the $\left[S U(3)_{c} \otimes S U(2)_{L} \otimes S U(2)_{R} \otimes U(1)_{X}\right]$ global symmetry of the composite sector, and are independent for each representation of massive fermions. Purely for technical simplicity, we further require these mass terms to be predominantly diagonal $3 \times 3$ matrices in generation space. That is, we are taking the composite Yukawa couplings to be the dominant source of breaking of generational $U(1)$ symmetries. Subdominantly, radiative corrections from Yukawa couplings will necessarily make the mass terms off-diagonal, but this will not play an important role.

\section{$5 \quad$ Partial Compositeness}

Partial compositeness is realized by adding a set of mass-mixing (soft mixing) terms to our model,

$$
\mathcal{L}_{\text {mixing }}=-M_{*}^{2} \frac{g_{e l}}{g_{*}} A_{\mu} \rho_{\mu}^{*}+\frac{M_{*}^{2}}{2}\left(\frac{g_{e l}}{g_{*}} A_{\mu}\right)^{2}+\left(\bar{\psi}_{L} \Delta \chi_{R}+\overline{\tilde{\psi}}_{R} \tilde{\Delta} \tilde{\chi}_{L}+\text { h.c. }\right),
$$

where an implicit sum over all species of gauge and fermionic fields is understood.

\subsection{Residual Standard Model Gauge Invariance}

The vector boson terms reflect the gauging of the global symmetry group of the composite sector of Section 4 by the elementary gauge symmetry. This looks somewhat unfamiliar because the (broken) $\rho$ gauge symmetry is being treated in unitary gauge. Indeed, it is 
straightforward to check that the entire Lagrangian, Eq. (1), is exactly invariant under an $S U(3) \otimes S U(2) \otimes U(1)$ gauge symmetry which we identify with the final SM gauge symmetry. The corresponding SM gauge fields are then superpositions

$$
\frac{g_{*}}{\sqrt{g_{e l}^{2}+g_{*}^{2}}} A_{\mu}+\frac{g_{e l}}{\sqrt{g_{e l}^{2}+g_{*}^{2}}} \rho_{\mu}^{*}
$$

and the SM gauge couplings have the form

$$
g=\frac{g_{e l} g_{*}}{\sqrt{g_{e l}^{2}+g_{*}^{2}}} \simeq g_{e l}, \quad \text { for } g_{e l} \ll g_{*} .
$$

The non-trivial superposition of $A_{\mu}$ and $\rho_{\mu}^{*}$ inside the SM gauge field is the vector meson version of partial compositeness. In the literature of low-energy hadronic phenomenology the analogous phenomenon is known as "photon- $\rho$ " mixing.

The fermionic terms in Eq. (22) describe fermionic partial compositeness in terms of mixing-mass parameters $\Delta, \tilde{\Delta}$, which are independent for each fermionic SM gauge-representation (or, equivalently, for each species of massive fermion). In the interest of simplicity, they are chosen to be diagonal in the same basis as the $m$ and $\tilde{m}$ are, in generation space. They explicitly break the separate elementary and composite gauge symmetries of the $\rho_{\mu}$ and $A_{\mu}$ but preserve the SM gauge invariance discussed above. The mass-mixing of elementary chiral fermions with composite Dirac fermions necessarily results in a new set of massless chiral fermions, which are linear combinations of the original $\chi$ and $\psi$, and are identified with the SM fermions.

\subsection{Mass Eigenstates}

Most phenomenological aspects of the model related to the production and detection of the new massive particles at the colliders are better pursued in the canonical language of diagonalized mass and kinetic terms. We diagonalize the mass mixing arising from $\mathcal{L}_{\text {mixing }}$ by field transformations:

$$
\begin{aligned}
& \left(\begin{array}{c}
A_{\mu} \\
\rho_{\mu}^{*}
\end{array}\right) \rightarrow\left(\begin{array}{cc}
\cos \theta & -\sin \theta \\
\sin \theta & \cos \theta
\end{array}\right)\left(\begin{array}{c}
A_{\mu} \\
\rho_{\mu}^{*}
\end{array}\right), \quad \tan \theta=\frac{g_{e l}}{g_{*}} \\
& \left(\begin{array}{c}
\psi_{L} \\
\chi_{L}
\end{array}\right) \rightarrow\left(\begin{array}{cc}
\cos \varphi_{\psi_{L}} & -\sin \varphi_{\psi_{L}} \\
\sin \varphi_{\psi_{L}} & \cos \varphi_{\psi_{L}}
\end{array}\right)\left(\begin{array}{c}
\psi_{L} \\
\chi_{L}
\end{array}\right), \quad \tan \varphi_{\psi_{L}}=\frac{\Delta}{m} \\
& \left(\begin{array}{c}
\tilde{\psi}_{R} \\
\tilde{\chi}_{R}
\end{array}\right) \rightarrow\left(\begin{array}{cc}
\cos \varphi_{\tilde{\psi}_{R}} & -\sin \varphi_{\tilde{\psi}_{R}} \\
\sin \varphi_{\tilde{\psi}_{R}} & \cos \varphi_{\tilde{\psi}_{R}}
\end{array}\right)\left(\begin{array}{c}
\tilde{\psi}_{R} \\
\tilde{\chi}_{R}
\end{array}\right), \quad \tan \varphi_{\tilde{\psi}_{R}}=\frac{\tilde{\Delta}}{\tilde{m}} .
\end{aligned}
$$

Our notation above has been chosen to be economical with symbols. Before the diagonalization, " $A_{\mu}, \psi_{L}, \tilde{\psi}_{R}$ " denoted the elementary fields and " $\rho_{\mu}^{*}, \tilde{\rho}_{\mu}, \chi, \tilde{\chi}$ " the composite fields. After the diagonalization, " $A_{\mu}, \psi_{L}, \tilde{\psi}_{R}$ " denote the SM fields, which are massless before EWSB, while " $\rho_{\mu}^{*}, \tilde{\rho}_{\mu}, \chi, \tilde{\chi}$ " denote new-physics mass eigenstates (before EWSB). 
The mixing angles relating the elementary/composite basis to the mass basis (before EWSB) now parametrize partial compositeness. Note that all the mixing angles are real and there is one for every SM multiplet,

$$
\begin{aligned}
\theta & \equiv \theta_{1}, \theta_{2}, \theta_{3} \\
\varphi_{\psi_{L}} & \equiv \varphi_{q_{L i}}, \varphi_{\ell_{L i}} \\
\varphi_{\tilde{\psi}_{R}} & \equiv \varphi_{u_{R i}}, \varphi_{d_{R i}}, \varphi_{\nu_{R i}}, \varphi_{e_{R i}} .
\end{aligned}
$$

The indices for $\theta$ refer to the $S U(3) \otimes S U(2) \otimes U(1)$ factors of the SM gauge group, while the ' $i$ ' indices on the $\varphi=\left\{\varphi_{\psi_{L}}, \varphi_{\tilde{\psi}_{R}}\right\}$ are generational.

\subsection{Diagonalized Lagrangian}

Implementing the transformation above in the total Lagrangian,

$$
\mathcal{L}=\mathcal{L}_{\text {gauge }}+\mathcal{L}_{\text {fermion }}+\mathcal{L}_{\text {Higgs }}
$$

gives:

$$
\begin{aligned}
& \mathcal{L}_{\text {gauge }}=-\frac{1}{4} F_{\mu \nu}^{2} \\
& +\frac{1}{2}\left(D_{\mu} \rho_{\nu} D_{\nu} \rho_{\mu}-D_{\mu} \rho_{\nu} D_{\mu} \rho_{\nu}\right)+\frac{M_{* 1}^{2}}{2} \tilde{\rho}_{\mu}^{2}+\frac{M_{*}^{2}}{2 \cos ^{2} \theta} \rho_{\mu}^{* 2}+\frac{i g}{2} F_{\mu \nu}\left[\rho_{\mu}, \rho_{\nu}\right] \\
& +2 i g \cot 2 \theta D_{\mu} \rho_{\nu}^{*}\left[\rho_{\mu}^{*}, \rho_{\nu}^{*}\right]+\frac{i g_{1}}{\sin \theta_{1}} D_{\mu} \tilde{\rho}_{\nu}\left[\tilde{\rho}_{\mu}, \tilde{\rho}_{\nu}\right] \\
& +i g_{1} \cot \theta_{1} D_{\mu} \rho_{\nu}^{*}\left[\tilde{\rho}_{\mu}, \tilde{\rho}_{\nu}\right]+i g_{1} \cot \theta_{1} D_{\mu} \tilde{\rho}_{\nu}\left(\left[\rho_{\mu}^{*}, \tilde{\rho}_{\nu}\right]+\left[\tilde{\rho}_{\mu}, \rho_{\nu}^{*}\right]\right) \\
& +\frac{g^{2}}{4}\left(\frac{\sin ^{4} \theta}{\cos ^{2} \theta}+\frac{\cos ^{4} \theta}{\sin ^{2} \theta}\right)\left[\rho_{\mu}^{*}, \rho_{\nu}^{*}\right]^{2}+\frac{g_{1}^{2}}{4 \sin ^{2} \theta_{1}}\left[\tilde{\rho}_{\mu}, \tilde{\rho}_{\nu}\right]^{2} \\
& +\frac{g_{1}^{2}}{4} \cot ^{2} \theta_{1}\left(\left[\rho_{\mu}^{*}, \tilde{\rho}_{\nu}\right]+\left[\tilde{\rho}_{\mu}, \rho_{\nu}^{*}\right]\right)^{2}+g_{1}^{2} \frac{\cos \theta_{1}}{\sin ^{2} \theta_{1}}\left[\tilde{\rho}_{\mu}, \tilde{\rho}_{\nu}\right]\left[\rho_{\mu}^{*}, \tilde{\rho}_{\nu}\right] \\
& \mathcal{L}_{\text {fermion }}=\bar{\psi}_{L} i \not D \psi_{L}+\bar{\chi}\left(i \not D-m_{*}\right) \chi \\
& +\bar{\psi}_{L}\left[g\left(\sin ^{2} \varphi_{\psi_{L}} \cot \theta-\cos ^{2} \varphi_{\psi_{L}} \tan \theta\right) \rho_{\mu}^{*}+\frac{g_{1}}{\sin \theta_{1}} \sin ^{2} \varphi_{\psi_{L}} \tilde{\rho}_{\mu}\right] \gamma^{\mu} \psi_{L} \\
& +\bar{\psi}_{L}\left(g \frac{\sin \varphi_{\psi_{L}} \cos \varphi_{\psi_{L}}}{\sin \theta \cos \theta} \rho_{\mu}^{*}+\frac{g_{1}}{\sin \theta_{1}} \sin \varphi_{\psi_{L}} \cos \varphi_{\psi_{L}} \tilde{\rho}_{\mu}\right) \gamma^{\mu} \chi_{L}+\text { h.c. } \\
& +\bar{\chi}_{L}\left[g\left(\cos ^{2} \varphi_{\psi_{L}} \cot \theta-\sin ^{2} \varphi_{\psi_{L}} \tan \theta\right) \rho_{\mu}^{*}+\frac{g_{1}}{\sin \theta_{1}} \cos ^{2} \varphi_{\psi_{L}} \tilde{\rho}_{\mu}\right] \gamma^{\mu} \chi_{L} \\
& +\bar{\chi}_{R}\left(g \cot \theta \rho_{\mu}^{*}+\frac{g_{1}}{\sin \theta_{1}} \tilde{\rho}_{\mu}\right) \gamma^{\mu} \chi_{R} \\
& +\left\{L \leftrightarrow R ; \chi \rightarrow \tilde{\chi} ; \varphi_{\psi_{L}} \rightarrow \varphi_{\tilde{\psi}_{R}} ; m_{*} \rightarrow \tilde{m}_{*}\right\}
\end{aligned}
$$




$$
\begin{aligned}
\mathcal{L}_{\text {Higgs }}=\mid & \left.D_{\mu} H\right|^{2}-V(H) \\
& +H^{\dagger} i g \cot \theta \rho_{\mu}^{*} D_{\mu} H+\text { h.c. } \\
& -i \frac{g_{1}}{2 \sin \theta_{1}}\left(\frac{1}{\sqrt{2}} \tilde{H}^{\dagger} \tilde{W}_{\mu}^{-} D_{\mu} H+\frac{1}{\sqrt{2}} H^{\dagger} \tilde{W}_{\mu}^{+} D_{\mu} \tilde{H}-\sqrt{\frac{2}{5}} H^{\dagger} \tilde{\mathcal{B}}_{\mu} D_{\mu} H\right)+\text { h.c. } \\
& -g_{1} g \frac{\cot \theta}{\sin \theta_{1}}\left(\frac{1}{\sqrt{2}} \tilde{H}^{\dagger} \rho_{\mu}^{*} \tilde{W}_{\mu}^{-} H+\frac{1}{\sqrt{2}} H^{\dagger} \rho_{\mu}^{*} \tilde{W}_{\mu}^{+} \tilde{H}-\sqrt{\frac{2}{5}} H^{\dagger} \rho_{\mu}^{*} \tilde{\mathcal{B}}_{\mu} H\right) \\
& +H^{\dagger}\left\{\left(g \cot \theta \rho_{\mu}^{*}\right)^{2}+\frac{g_{1}^{2}}{\sin ^{2} \theta_{1}}\left(\frac{1}{2} \tilde{W}_{\mu}^{+} \tilde{W}_{\mu}^{-}+\frac{1}{10} \tilde{\mathcal{B}}_{\mu}^{2}\right)\right\} H \\
& -\left(\sin \varphi_{\psi_{L}} \bar{\psi}_{L}+\cos \varphi_{\psi_{L}} \bar{\chi}_{L}\right)\left[Y_{* u} \tilde{H}\left(\sin \varphi_{\tilde{\psi}_{R}^{u}} \tilde{\psi}_{R}^{u}+\cos \varphi_{\tilde{\psi}_{R}^{u}} \tilde{\chi}_{R}^{u}\right)\right. \\
& \left.\quad+Y_{* d} H\left(\sin \varphi_{\tilde{\psi}_{R}^{d}} \tilde{\psi}_{R}^{d}+\cos \varphi_{\tilde{\psi}_{R}^{d}} \tilde{\chi}_{R}^{d}\right)\right]+ \text { h.c. } \\
& -\bar{\chi}_{R} Y_{* u} \tilde{H}_{\chi_{L}^{u}}^{u}-\bar{\chi}_{R} Y_{* d} H \tilde{\chi}_{L}^{d}+\text { h.c. },
\end{aligned}
$$

where $\tilde{\psi}_{R}^{u} \equiv\left\{u_{R}, \nu_{R}\right\}, \tilde{\psi}_{R}^{d} \equiv\left\{d_{R}, e_{R}\right\}$, and, we recall, $\tilde{\chi}^{u} \equiv\{\tilde{U}, \tilde{N}\}, \tilde{\chi}^{d} \equiv\{\tilde{D}, \tilde{E}\}$. In Eqs. (30), (31) and (32), all covariant derivatives of fermions and heavy gauge bosons are now with respect to the unbroken SM gauge group,

$$
D_{\mu} \equiv \partial_{\mu}-i g A_{\mu}, \quad g=g_{*} \sin \theta
$$

and

$$
m_{*} \equiv \sqrt{\Delta^{2}+m^{2}}, \quad \tilde{m}_{*} \equiv \sqrt{\tilde{\Delta}^{2}+\tilde{m}^{2}} .
$$

All vector fields in Eqs. (31) and (32), (including those in the covariant derivatives) are to be considered in a matrix notation, i.e. each gauge component multiplies its corresponding generator $T^{a}$, normalized according to the standard convention $\left(\operatorname{Tr}\left(T^{a} T^{b}\right)=\delta^{a b} / 2\right.$ for the non-abelian generators). The only exception is for $\tilde{W}^{ \pm}$and $\tilde{\mathcal{B}}$ in Eq. (32), which are component fields. This complication arises as a result of the subtle transformation of the Higgs under $\left[S U(2)_{R}\right]^{\text {comp }}$. In the gauge Lagrangian (30) we adopt a different notation: gauge fields are still in matrix notation, with an implicit trace operation over the whole Lagrangian, but with the following normalization: $\operatorname{Tr}\left(T^{a} T^{b}\right)=\delta^{a b}$ for non-abelian generators, $T=1$ for the abelian ones. This choice leads to a more compact and simple-to-read expression, compared to the standard normalization or the use of component fields. Gauge couplings, mixing angles and masses of the heavy gauge bosons without explicit indices must be understood as collective symbols (see Eq. (28) as well): 8

$$
g=\left\{g_{3}, g_{2}, g_{1}\right\}, \quad M_{*}=\left\{M_{* 3}, M_{* 2}, M_{* 1}\right\}
$$

and similarly for the masses and Yukawa couplings of the heavy fermions (see also Eq. (21)):

$$
m_{*} \equiv\left\{m_{*}^{Q}, m_{*}^{L}\right\}, \quad \tilde{m}_{*} \equiv\left\{\tilde{m}_{*}^{U}, \tilde{m}_{*}^{D}, \tilde{m}_{*}^{N}, \tilde{m}_{*}^{E}\right\}
$$

\footnotetext{
${ }^{8}$ As declared in Section 4.1, we are assuming for simplicity a common mass parameter $M_{* 1}$ for $\tilde{W}^{ \pm}, \tilde{\mathcal{B}}$, and $\mathcal{B}^{*}$.
} 
With the definitions of the collective symbols of the fields, Eqs. (15), (19) and (20), all terms in the action after mass diagonalization can be easily decoded by summing over the implicit indices.

The effective field theory (EFT) of Eqs. (30)-(32) describes the SM field content plus a set of heavy gauge and fermionic excitations. Its only exact gauge invariance is that of the SM gauge symmetry, as one can easily check by noticing that the SM gauge fields couple to the heavy fields only through SM covariant derivatives and field strengths. The (leading) terms of the SM Lagrangian, interpreted as the low-energy effective limit of the Lagrangian (29) below $m_{*}, \tilde{m}_{*}, M_{*}$, then follow by setting to zero all terms in Eq. (29) that involve the heavy fields. The SM Yukawa couplings, $Y$, are written in terms of the composite Yukawa couplings, $Y_{*}$, as follows:

$$
\begin{array}{rlrl}
\left(Y_{u}\right)_{i j} & =\sin \varphi_{q_{L i}}\left(Y_{* U}\right)_{i j} \sin \varphi_{u_{R j}} & & \left(Y_{\nu}\right)_{i j}=\sin \varphi_{\ell_{L i}}\left(Y_{* N}\right)_{i j} \sin \varphi_{\nu_{R j}} \\
\left(Y_{d}\right)_{i j}=\sin \varphi_{q_{L i}}\left(Y_{* D}\right)_{i j} \sin \varphi_{d_{R j}}, & \left(Y_{e}\right)_{i j}=\sin \varphi_{\ell_{L i}}\left(Y_{* E}\right)_{i j} \sin \varphi_{e_{R j}},
\end{array}
$$

where there is no sum on repeated indices. Note that the SM Yukawas are off-diagonal and $h i$ erarchical, while those before the mass diagonalization are off-diagonal and non-hierarchical. Using Eq. (37), the mass-diagonalized Lagrangian (29) can be written in terms of all the SM parameters (gauge and Yukawa couplings plus the Higgs quartic coupling and mass term), as well as mixing angles for each SM particle and heavy-physics mass scales, $m_{*}, \tilde{m}_{*}, M_{*}^{2}$.

\subsection{Parameter Space Beyond the Standard Model}

Now that we have presented the diagonalized Lagrangian containing the SM and a set of heavy fields with which the SM particles interact, we comment briefly on the extended parameter space of the model. We began with a model described by the theoretical parameter space of the elementary and composite sectors, $\left\{g_{e l}, g_{*}, Y_{*}, m, \tilde{m}, \Delta, \tilde{\Delta}, M_{*}, \lambda_{H}, \mu_{H}\right\}$. After diagonalizing, we are left with a Lagrangian written in terms of the parameters of the Standard Model, $\left\{g, Y, \lambda_{H}, \mu_{H}\right\}$, plus a set of mixing angles $\{\theta, \varphi, \tilde{\varphi}\}$ and masses of the new heavy states $\left\{m_{*}, \tilde{m}_{*}, M_{*}\right\}$. Thus, we can think of the mixing angles and the heavy masses as new parameters which can be varied to explore the parameter space subject to the constraints:

$$
\begin{gathered}
g=g_{*} \sin \theta \\
\left(Y_{S M}\right)_{i j}=\sin \varphi_{\psi_{L i}}\left(Y_{*}\right)_{i j} \sin \varphi_{\psi_{R j}}
\end{gathered}
$$

and our assumption that the composite sector is more strongly coupled than the elementary sector:

$$
g_{*}, Y_{*} \sim 1-4
$$

\section{EWSB}

After EWSB, mass terms proportional to the Higgs vev mix different states, and a further diagonalization is needed. In the electroweak unitary gauge, the charged and neutral gauge mass matrices are: 


$$
\begin{aligned}
& W_{\mu}^{+} \quad W_{\mu}^{*+} \quad \tilde{W}_{\mu}^{+} \\
& M_{ \pm}^{2}=\frac{v^{2}}{4}\left(\begin{array}{ccc}
g_{2}^{2} & g_{2}^{2} \frac{c_{2}}{s_{2}} & -g_{1} g_{2} \frac{1}{s_{1}} \\
g_{2}^{2} \frac{c_{2}}{s_{2}} & \frac{4 M_{* 2}^{2}}{c_{2}^{2} v^{2}}+g_{2}^{2} \frac{c_{2}^{2}}{s_{2}^{2}} & -g_{1} g_{2} \frac{c_{2}}{s_{1} s_{2}} \\
-g_{1} g_{2} \frac{1}{s_{1}} & -g_{1} g_{2} \frac{c_{2}}{s_{1} s_{2}} & \frac{4 M_{* 1}^{2}}{v^{2}}+g_{2}^{2} \frac{1}{s_{2}^{2}}
\end{array}\right) W_{\mu}^{-} W_{\mu}^{*-} \tilde{W}_{\mu}^{-} \\
& \begin{array}{ccccc}
W_{\mu}^{3} & \mathcal{B}_{\mu} & W_{\mu}^{* 3} & \mathcal{B}_{\mu}^{*} & \tilde{\mathcal{B}}_{\mu}
\end{array} \\
& M_{0}^{2}=\frac{v^{2}}{4}\left(\begin{array}{ccccc}
g_{2}^{2} & -g_{Y} g_{2} & g_{2}^{2} \frac{c_{2}}{s_{2}} & -g_{Y} g_{2} \frac{c_{1}}{s_{1}} & -g_{Y} g_{2} \frac{1}{s_{1}} \sqrt{\frac{2}{3}} \\
-g_{Y} g_{2} & g_{Y}^{2} & -g_{Y} g_{2} \frac{c_{2}}{s_{2}} & g_{Y}^{2} \frac{c_{1}}{s_{1}} & g_{Y}^{2} \frac{1}{s_{1}} \sqrt{\frac{2}{3}} \\
g_{2}^{2} \frac{c_{2}}{s_{2}} & -g_{Y} g_{2} \frac{c_{2}}{s_{2}} & \frac{4 M_{* 2}^{2}}{c_{2}^{2} v^{2}}+g_{2}^{2} \frac{c_{2}^{2}}{s_{2}^{2}} & -g_{Y} g_{2} \frac{c_{1} c_{2}}{s_{1} s_{2}} & -g_{Y} g_{2} \frac{c_{2}}{s_{1} s_{2}} \sqrt{\frac{2}{3}} \\
-g_{Y} g_{2} \frac{c_{1}}{s_{1}} & g_{Y}^{2} \frac{c_{1}}{s_{1}} & -g_{Y} g_{2} \frac{c_{1} c_{2}}{s_{1} s_{2}} & \frac{4 M_{* 1}^{2}}{c_{1}^{2} v^{2}}+g_{Y}^{2} \frac{c_{1}^{2}}{s_{1}^{2}} & g_{Y}^{2} \frac{c_{1}}{s_{1}^{2}} \sqrt{\frac{2}{3}} \\
-g_{Y} g_{2} \frac{1}{s_{1}} \sqrt{\frac{2}{3}} & g_{Y}^{2} \frac{1}{s_{1}} \sqrt{\frac{2}{3}} & -g_{Y} g_{2} \frac{c_{2}}{s_{1} s_{2}} \sqrt{\frac{2}{3}} & g_{Y}^{2} \frac{c_{1}}{s_{1}^{2}} \sqrt{\frac{2}{3}} & \frac{4 M_{* 1}^{2}}{v^{2}}+g_{Y}^{2} \frac{1}{s_{1}^{2}} \sqrt{\frac{2}{3}}
\end{array}\right) W_{\mu}^{* 3} \mathcal{B}_{\mu}
\end{aligned}
$$

where $v=246 \mathrm{GeV}, s_{1,2} \equiv \sin \theta_{1,2}, c_{1,2} \equiv \cos \theta_{1,2}$, and we have expressed Eq. (42) in terms of $g_{Y}=\sqrt{3 / 5} g_{1}$ instead of $g_{1}$ to have a more easy-to-read expression. The fermionic mass matrices for up and down states are:

$$
\begin{aligned}
& u_{L} \quad U_{L} \quad \tilde{U}_{L} \\
& M_{U}=\left(\begin{array}{ccc}
Y_{u} \frac{v}{\sqrt{2}} & \frac{c}{s} Y_{u} \frac{v}{\sqrt{2}} & 0 \\
0 & m_{*}^{Q} & s^{-1} Y_{u} \tilde{s}_{u}^{-1} \frac{v}{\sqrt{2}} \\
Y_{u} \frac{\tilde{c}_{u}}{\tilde{s}_{u}} \frac{v}{\sqrt{2}} & \frac{c}{s} Y_{u} \frac{\tilde{c}_{u}}{\tilde{s}_{u}} \frac{v}{\sqrt{2}} & \tilde{m}_{*}^{U}
\end{array}\right) u_{R} \\
& d_{L} \quad D_{L} \quad \tilde{D}_{L} \\
& M_{D}=\left(\begin{array}{ccc}
Y_{d} \frac{v}{\sqrt{2}} & \frac{c}{s} Y_{d} \frac{v}{\sqrt{2}} & 0 \\
0 & m_{*}^{Q} & s^{-1} Y_{d} \tilde{s}_{d}^{-1} \frac{v}{\sqrt{2}} \\
Y_{d} \frac{\tilde{c}_{d}}{\tilde{s}_{d}} \frac{v}{\sqrt{2}} & \frac{c}{s} Y_{d} \frac{\tilde{c}_{d}}{\tilde{s}_{d}} \frac{v}{\sqrt{2}} & \tilde{m}_{*}^{D}
\end{array}\right) d_{R}
\end{aligned}
$$


where we have defined $s \equiv \sin \varphi_{u_{L}}, c \equiv \cos \varphi_{u_{L}} ; \tilde{s}_{u, d} \equiv \sin \varphi_{u_{R}, d_{R}}, \tilde{c}_{u, d} \equiv \cos \varphi_{u_{R}, d_{R}}$, and $Y_{u}$, $Y_{d}$ are the up- and down-type SM Yukawa couplings defined by Eq. (37).

In general it is best to proceed numerically for any choice of model parameters in diagonalizing the above mass matrices, and rewriting the Lagrangian of Eq. (29) in terms of the resultant mass-eigenstate fields, thereby obtaining the final interaction vertices for the bottom-line mass eigenstates. In the case of the gauge mass matrices, however, a reasonable approximation consists in treating the EWSB corrections as small perturbations, and work at leading order. This is consistent since the EWSB terms are smaller than the mass splitting in the heavy-heavy sector. In the fermionic mass matrices this is not true in general, and a full diagonalization is therefore needed. A further complication comes from the fact that each of the elements of $M_{U}, M_{D}$ is actually a $3 \times 3$ matrix in flavor space. Depending on the flavor structure of the composite Yukawa matrices $Y_{*}$, which in turn must combine with the $\sin \varphi$ to yield realistic SM Yukawa couplings, a full diagonalization might be needed only generation by generation, or worst case for the whole $9 \times 9$ fermionic matrices. The first possibility occurs if $Y_{*}$ is approximately diagonal in flavor space, maybe as the result of a flavor symmetry of the composite sector. No simplification is instead possible in the opposite, extreme case of anarchic $Y_{*}$, that is if all entries of $Y_{*}$ are of the same order and large. Quite interestingly, these two different realizations of flavor could be experimentally distinguishable at future colliders. We will discuss this in Section 10.

\section{$7 \quad$ Full $t_{R}$ Compositeness}

We have already said that the size of the SM Yukawa couplings is controlled, in our model, by the size of the composite Yukawas $Y_{*}$ and by the degree of compositeness of the fermion mass eigenstates, Eq. (37). In the particular case of the top quark, we have:

$$
Y_{\text {top }}=\sin \varphi_{t_{L}}\left(Y_{* U 33}\right) \sin \varphi_{t_{R}}
$$

Simple inspection of this formula shows that the (large) SM top Yukawa coupling can be reproduced only if the mixing angles of $t_{R}$ and $t_{L}$ are not both too small. We are assuming that $\left(Y_{* U 33}\right)$ is not too strong, say $Y_{* U 33} \lesssim 3$, both to stay in theoretical control of the composite sector and also because the electroweak $T$ parameter scales as $\left(Y_{* U 33}\right)^{4}$. Now the

mixing angles control the partial compositeness phenomenology of SM particles, yielding deviations from SM predictions. While present top quark tests pose no conflict with sizeable mixing angles, note that the electroweak symmetry implies

$$
\varphi_{b_{L}}=\varphi_{t_{L}}
$$

and the couplings of $b_{L}$ are very well tested, in particular its coupling to the $Z, g_{L b}$. Large values of the mixing angle $\varphi_{b_{L}}$ imply a sizable correction $\delta g_{L b}$ to the $Z b \bar{b}$ vertex, as the calculation of Appendix C explicitly shows.

The best one can imagine in easing the tension between the constraint on $\delta g_{L b}$ and Eq. (45) is to satisfy the latter with the smallest possible $\varphi_{t_{L}}$ and the largest possible $\varphi_{t_{R}}$. This has an immediate cost, in that loops involving the elementary $t_{R}$, such as Fig. 1, 


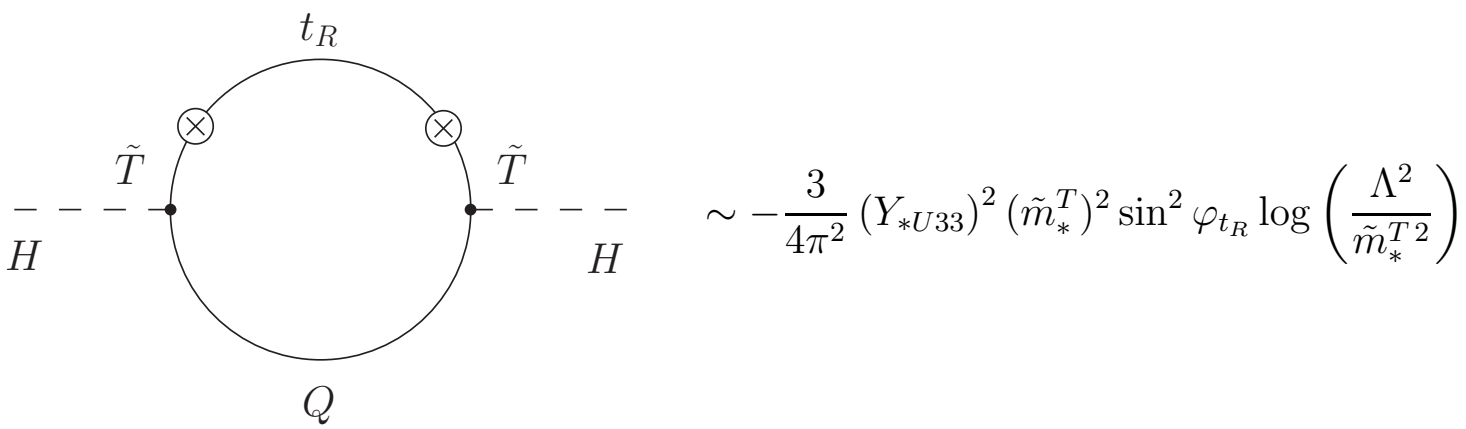

Figure 1: One-loop log divergent contribution to the Higgs mass squared from the virtual exchange of an elementary $t_{R}$. A circled cross denotes a $\Delta_{t_{R}}$ mass mixing. We used Eqs. (27), (34) to set $\Delta_{t_{R}}=\tilde{m}_{*}^{T} \sin \varphi_{t_{R}}$, and the fact that additional $\Delta_{t_{R}}$ insertions on the $t_{R}$ elementary propagator are suppressed for divergent virtual momenta.

strongly correct the Higgs mass squared, with composite-strength couplings and without mixing-angle suppression. For $m_{*}>\mathrm{TeV}$, this implies a Higgs fine tuning at the 10 percent level or worse. This problem reflects a breakdown in our philosophy. For $\sin \varphi_{t_{R}} \sim \mathcal{O}(1)$, the separation between an elementary sector weakly coupled to (and weakly mixed with) a stronger composite sector is lost.

These problems suggest a different scenario. Rather than thinking of $t_{R}$ as an elementary field strongly coupled to the composites, it makes better sense to posit that the $t_{R}$ is itself a full chiral member of the composite sector (with no elementary admixture), and its participation in the composite dynamics is an integral part of generating a light Higgs multiplet. 9 From this viewpoint, quantum loops involving only the $t_{R}$ and other composites, Fig. 1, do not contribute to our measure of fine tuning, as discussed in subsection 4.2. With the $t_{R}$ now a full composite, the remaining elementary particles have reasonably small mixing angles suppressing radiative corrections to the Higgs mass squared, resulting in mild tuning.

This is the physical picture we will assume from now on. The question is how to amend our present construction to take this into account. We do this by continuing to use Eq. (29) to pursue the phenomenological implications of the model, but we simply take a composite- $t_{R}$ limit,

$$
\sin \varphi_{t_{R}}=1 \text {. }
$$

As discussed above, Higgs fine tuning is then only measured with respect to sensitivity to mixing angles of the remaining elementary fields.

While Nature might have even heavier composites with the quantum numbers of the light Higgs, they are not required in our minimal model, and we have not included them. Similarly, now that $t_{R}$ is a complete composite, which we allow to interact directly with the Higgs before the diagonalization of Section 5, we will no longer need to retain the heavy Dirac composite excitation with the same quantum numbers, $\tilde{T}$, in the minimal model (although again Nature may possess such a state). A minor extra payoff of this is that the dominant contributions

\footnotetext{
${ }^{9}$ This now means that SM gauge anomalies cancel non-trivially between the new elementary and new composite sectors.
} 
to fine tuning in the Higgs mass will turn out to be UV-finite, that is there are not even logarithmic divergences. Again, we can continue to utilize Eq. (29) for phenomenological investigation, but with the second limit in which we throw away terms containing $\tilde{T}$ :

$$
\tilde{T} \rightarrow 0
$$

In performing low-momentum calculations, we find it useful to work with the lagrangian before elementary/composite diagonalization, Eqs. (11), (13) and (22). The equivalent $t_{R^{-}}$ compositeness limit in this language is given by

$$
\tilde{T}_{L} \rightarrow 0, \quad \tilde{T}_{R} \rightarrow t_{R}
$$

\section{Higgs Fine Tuning and Finiteness}

Let us discuss more in detail about the Higgs fine tuning in the minimal model with composite $t_{R}$. As anticipated in the overview, we choose as a measure of tuning the ratio of the desired Higgs mass squared, $m_{h}^{2}$, to the largest 1-loop correction sensitive to the mixing with the elementary sector,

$$
\text { Fine tuning } \sim \frac{m_{h}^{2}}{\delta m_{h \mid \text { mixing }}^{2}} .
$$

Note that $m_{h}$ denotes the physical Higgs boson mass, not the mass parameter of the Higgs doublet. The 1-loop correction to the Higgs mass squared from the composite sector alone is assumed to be reasonably well subsumed into the parameters of the tree-level potential, $V(H)$, and by assumption does not contribute to fine tuning, as discussed in subsection 4.2.

The corrections $\delta m_{h \mid \text { mixing }}^{2}$ from gauge and fermionic fields are most easily computed in the elementary/composite basis. The relevant Feynman diagrams are those of Fig. 2 , This choice of basis also makes manifest the central mechanism for cutting off the large quadratic divergences: the elementary fields only couple to the Higgs via their mass mixing with the composite fields, and this implies enough propagators to make the loop integral convergent. Technically, this good behavior reflects the collective-breaking structure of our model. Physically, it captures the UV form-factor suppression due to partial compositeness.

The computation of the diagrams of Fig. 2 is straightforward, with the only subtlety of resumming all possible elementary/composite mass insertions on the elementary propagator. In the case of the elementary $t_{L}$ field, the effect of this resummation is that of modifying the propagator to:

$$
\frac{i}{\not p} \longrightarrow \frac{i}{\not p} \cdot \frac{1}{1-\frac{\Delta_{t_{L}}^{2}}{p^{2}-\left(m^{Q}\right)^{2}}} .
$$

The resummed elementary gauge propagator is instead

$$
\frac{-i}{p^{2}} \cdot \frac{1}{1-\frac{M_{*}^{2} \tan ^{2} \theta}{p^{2}-M_{*}^{2}}}\left(\eta_{\mu \nu}-\frac{p_{\mu} p_{\nu}}{p^{2}}\right)
$$



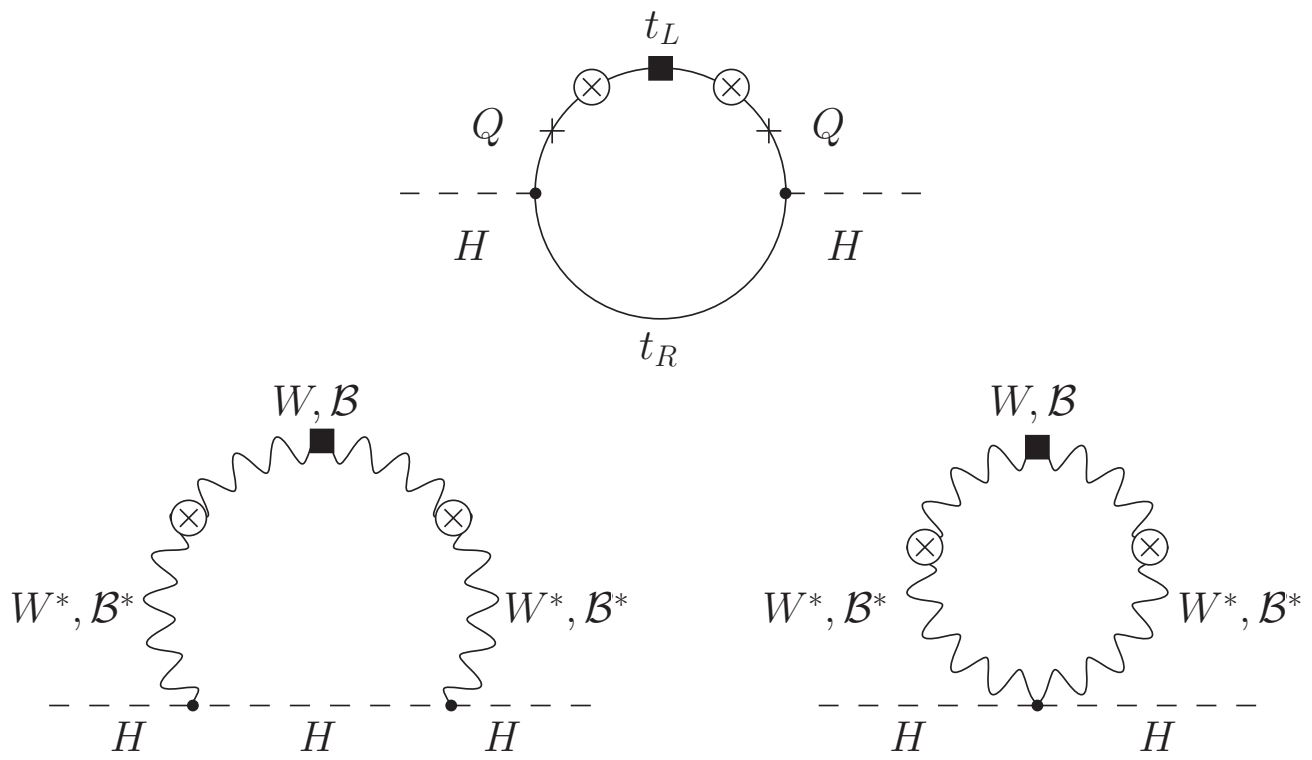

Figure 2: One-loop corrections to the Higgs mass squared in the minimal model with composite $t_{R}$ adopting the elementary/composite basis. Black squares represent resummation of all higher elementary/composite mass-mixing.

while the (un-resummed) $W^{*}, B^{*}$ propagator to be used to compute the gauge diagrams of Fig. 2 is the usual one for a vector of mass $M_{* 1}, M_{* 2}$. We thus obtain, respectively for the gauge and fermion contribution to the physical Higgs mass squared (after rotating to the Euclidean),

$$
\begin{aligned}
& \delta m_{h \mid \text { gauge }}^{2}=-\frac{9 g_{2}^{2}}{16 \pi^{2}} \int_{0}^{\infty} d p p \cdot \mathcal{F}_{\text {gauge }}\left(p^{2}, \theta_{2}, M_{* 2}\right)+\frac{1}{3} \cdot \frac{3}{5}\left(\theta_{2}, g_{2}, M_{* 2} \leftrightarrow \theta_{1}, g_{1}, M_{* 1}\right) \\
& \mathcal{F}_{\text {gauge }}\left(p^{2}, \theta_{2}, M_{*}\right)=\frac{M_{*}^{4} / \cos ^{2} \theta_{2}}{\left(p^{2}+M_{*}^{2}\right)\left(p^{2}+M_{*}^{2} / \cos ^{2} \theta_{2}\right)} \\
& \delta m_{h \mid \text { fermion }}^{2}=\frac{3}{2 \pi^{2}} Y_{\text {top }}^{2} \int_{0}^{\infty} d p p \cdot \mathcal{F}_{\text {fermion }}\left(p^{2}, \varphi_{t_{L}}, m_{* 33}^{Q}\right) \\
& \mathcal{F}_{\text {fermion }}\left(p^{2}, \varphi_{t_{L}}, m_{* 33}^{Q}\right)=\frac{\left(m_{* 33}^{Q}\right)^{4} \cos ^{2} \varphi_{t_{L}}}{\left(p^{2}+\left(m_{* 33}^{Q}\right)^{2} \cos ^{2} \varphi_{t_{L}}\right)\left(p^{2}+\left(m_{* 33}^{Q}\right)^{2}\right)}
\end{aligned}
$$

All formulas have been expressed in terms of physical mass parameters and mixing angles, and we have neglected the tree-level Higgs mass for simplicity. The factor of $3 / 5$ in Eq. (53) is due to our normalization of the hypercharge. The form factors $\mathcal{F}_{\text {fermion }}, \mathcal{F}_{\text {gauge }}$ cut off the quadratic divergences for virtual momenta much larger than the mass of the heavy excitations; setting them to 1 gives the divergent SM results. By approximating $\cos \varphi_{t_{L}}=1$, 
$\cos \theta_{1,2}=1$ in the form factors, one obtains

$$
\delta m_{h \mid \text { gauge }}^{2} \simeq-\frac{3}{32 \pi^{2}}\left(3 g_{2}^{2} M_{* 2}^{2}+g_{Y}^{2} M_{* 1}^{2}\right), \quad \delta m_{h \mid \text { fermion }}^{2} \simeq \frac{3}{2 \pi^{2}} \frac{m_{t}^{2}}{v^{2}}\left(m_{* 33}^{Q}\right)^{2} .
$$

\section{$9 \quad$ Viable Parameter Space}

While the leading terms in the SM EFT below $m_{*}, M_{*}$ are given by simply setting to zero all heavy fields in Eq. (29), the body of precision electroweak and flavor data also constrains higher-dimension operators obtained by more carefully integrating out the heavy physics. The most important constraints come from oblique corrections encoded in the Peskin-Takeuchi $S$ and $T$ parameters [42], modifications of the $Z b_{L} \bar{b}_{L}$ coupling, $\delta g_{L b}$, and flavor-changing four-fermion operators. The leading contributions to $S, T$ and $\delta g_{L b}$ due to the exchange of the heavy resonances are computed in the Appendix, Eqs. (71), (73) and (77). These simple formulas can be used to identify the viable parameter space, determining thereby where and how new physics can be produced explicitly in collider searches. Flavor physics experiments constrain the parameter space in more complex ways, sensitive to $Y_{* U, D}$, and can be neglected in a simple, model-independent analysis. Broadly speaking, there is a generalized GIM mechanism saving the model from catastrophic failure in regard to flavorchanging rare processes, which is illustrated in Appendix A. Various dedicated analyses have been carried out in the context of full higher-dimensional warped models [6]. A rough conclusion appears to be that $M_{*}$ should be above roughly $2 \mathrm{TeV}$, in particular $M_{* 3}$, in order for reasonable flavor ansatze to survive. This agrees and is consistent with the constraints imposed by the electroweak observables, which we now want to discuss in some detail 10

Let us make the simplifying hypothesis of universal masses $M_{*}, m_{*}$ and composite couplings $g_{*}$. Equations (71), (73) and (77) then express the three precision observables $S, T$ and $\delta g_{L b}$ as functions of $m_{*}, M_{*}, Y_{* U 33}, Y_{* D 33}$, and $g_{*}$. 11 For definiteness, we set $g_{*}=3$ and vary the remaining parameters. The body of precision data (see for example [43, 44]) can then be used to constrain the plane $\left(m_{*}, M_{*}\right)$ for fixed $Y_{* U 33}$ and $Y_{* D 33}$, provided the Higgs mass $m_{h}$ is also specified. The results are shown in Fig. 3, where we used $m_{h}=250 \mathrm{GeV} .12$

One can see that there is a sizable portion of parameter space that is not ruled out and that should be accessible at the LHC, as we will discuss more in detail in the next section. The constraint is stronger on $M_{*}$, mainly due to the $S$ parameter, while the fermion mass $m_{*}$ can be lighter. Lighter masses for the fermionic resonances are also preferred to keep

${ }^{10}$ Precision electroweak effects are but a virtual shadow of new physics, which can easily be sensitive to modest non-minimal physics which we cannot anticipate in advance. Therefore in collider searches for new physics, precision data should be consulted to give a ballpark guide to the viable parameter space, rather than trusting the sharp boundaries that emerge in analysing any particular model. For example, the constraint from $Z b \bar{b}$ can be strongly relaxed if the $S U(2)_{L} \times S U(2)_{R}$ symmetry of the composite sector is enlarged to include a discrete LR parity, and additional composite states are added [11.

${ }^{11}$ We use the formula of the top Yukawa coupling, Eq. (45), to express $\sin \varphi_{t_{L}}$ in terms of $Y_{* U 33}$, and hence $m_{33}^{Q}$ in terms of $m_{* 33}^{Q}$ and $Y_{* U 33}$.

${ }^{12}$ In detail, we perform a $\chi^{2}$ test using the fit to $S, T$ and $\delta g_{L b}$ of Ref. [10] with $m_{h}=250 \mathrm{GeV}$, and impose a $99 \%$ CL bound. 

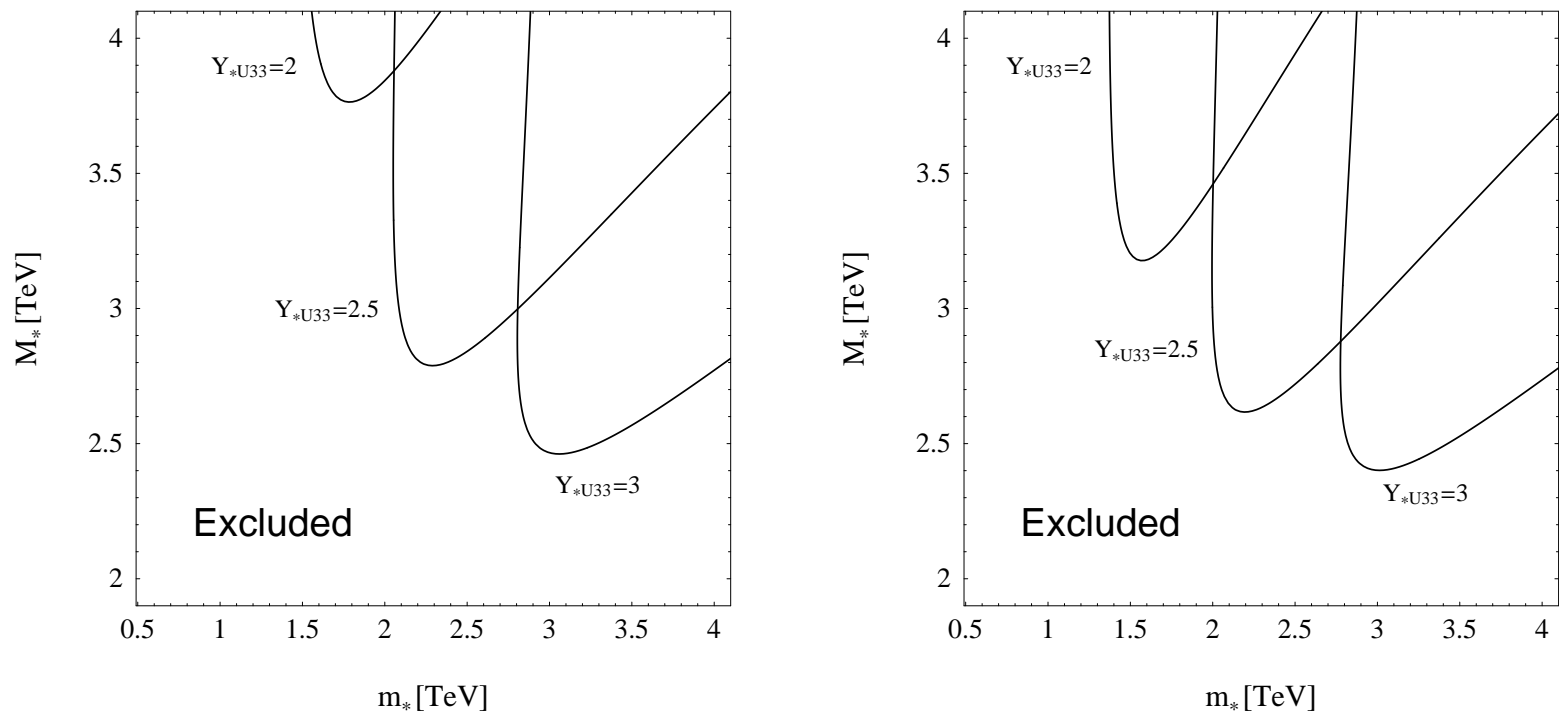

Figure 3: Exclusion curves in the plane $\left(m_{*}, M_{*}\right)$ for $Y_{* U 33}=2,2.5,3$ and $Y_{* D 33} / Y_{* U 33}=1 / 2$ (left plot) or $Y_{* D 33} / Y_{* U 33}=1 / 3$ (right plot). The area below each curve is excluded at $99 \%$ $C L$ by a combined fit to the electroweak observables $S, T$ and $\delta g_{L b}$.

the residual fine tuning in the Higgs mass small. The estimate of Eq. (50), together with Eq. (57), shows that the level of cancellation in the one-loop correction to the Higgs mass squared, required to obtain $m_{h}=250 \mathrm{GeV}$, is not worse than $\sim 10 \%$ for $m_{*}$ up to $3 \mathrm{TeV}$. This means that the viable region of Fig. 3 is also a natural place for the parameters of our model.

\section{Phenomenology Highlights and Challenges}

The minimal scenario of partial compositeness that emerges from the electroweak precision tests is particularly challenging for the LHC. Vector excitations are to be at $2-3 \mathrm{TeV}$ or heavier, while fermions can be lighter, as also suggested by fine-tuning considerations. The new states are expected to couple strongly to the third-generation SM quarks, but weakly to the light fermions. This makes them difficult to discover at a hadron collider, though there are specific processes that are particularly promising.

Partial compositeness predicts a quite well determined pattern of new physics at the LHC, whose key features are robust and do not depend on the details of the models. Perhaps the most relevant exception to this rule comes in the phenomenology of the heavy fermions, where two qualitatively different scenarios can arise, depending on whether flavor mixing effects in the composite sector are large or not. We will start by considering first the case in which flavor-changing effects in the composite Yukawa matrices $Y_{*}$ are small, and then see how the phenomenology changes by relaxing this hypothesis. 


\subsection{Heavy gauge boson production and decays}

The most effective strategy to discover the new particles can be deduced by looking at the magnitude of their couplings in the Lagrangian (29). Heavy excitations of the SM gauge bosons, $\rho^{*}$, couple to the SM fermions with strength

$$
g\left(\sin ^{2} \varphi \cot \theta-\cos ^{2} \varphi \tan \theta\right)
$$

as one can see from the second line of Eq. (31). In the case of light SM fermions the first term is highly suppressed, since $\varphi \ll 1$, and the whole interaction is accounted for by the second term. The latter is due to the (universal) $A-\rho$ mixing and it is still suppressed by a factor $\tan \theta \simeq\left(g / g_{*}\right)$ compared to SM couplings. By contrast, third-generation quarks have a large mixing angle $\varphi$ and the first term dominates. The coupling of $\rho^{*}$ to the longitudinal polarizations of the SM weak bosons is also strong, the latter being composite degrees of freedom. It can be extracted, in the unphysical basis and using the Equivalence theorem, from the second line of Eq. (32): $g \cot \theta \simeq g_{*}$. Producing and detecting the heavy vectors will be challenging for the LHC due to their small couplings to light fermions. Single production mainly proceeds via Drell-Yan scattering

$$
q \bar{q} \longrightarrow \rho^{*}
$$

Electroweak heavy excitations $W^{* \pm}, W^{* 3}, \mathcal{B}^{*} 13$ can also be produced via weak boson fusion, as in technicolor theories. Despite the large coupling to longitudinal $W$ 's and $Z$ 's, this process is subdominant for large masses $M_{*} \gtrsim 2-3 \mathrm{TeV}$, as well as other production mechanisms like gluon fusion, $t \bar{t}$ and $t \bar{b}$ associated productions. 14 Once produced, the heavy vectors will mainly decay to pairs of third-generation SM quarks, $t \bar{t}, b \bar{b}$ or $t \bar{b}$, and pairs of longitudinally polarized SM vector bosons, $Z_{L} h, W_{L}^{+} W_{L}^{-}$or $W_{L}^{ \pm} Z_{L}$. Decays to light SM quarks and leptons will instead be rare. When kinematically allowed, also decays to one SM top or bottom plus one excited top or bottom quark (TE, $T \bar{b}$ etc.), or even to any two heavy fermions will be important, thanks to their large couplings to the excited vectors (first terms of third and fourth lines in Eq. (31)).

In the case of the electroweak heavy vectors $W^{* \pm}, W^{* 3}, \mathcal{B}^{*}$, it is very useful to compare our scenario with Little Higgs (LH) models, for which numerous and detailed studies exist in the literature [45, 46, 47, 48, 49]. In fact, the structure of the gauge sector of LH models based on product groups (and without T-parity) is similar to that prescribed by partial compositeness: the product of two $S U(2) \times U(1)$ groups is broken down spontaneously to the diagonal subgroup (the latter being identified with the SM symmetry), and the SM fermions transform under only the first gauge group. This is analogous to the elementary/composite mixing of sec. 5, considering that the SM light fermions are almost completely elementary, and as such their couplings to the composite gauge bosons are strongly suppressed. In particular, the mixing angles between the two $S U(2) \times U(1)$ groups of the LH theories, $\theta_{L H}$,

\footnotetext{
${ }^{13}$ Here and in the following we classify the new vectors using their $S U(2)_{L}$ quantum numbers, since the EWSB effects are small, see section 6 .

${ }^{14} \mathrm{~K}$.Agashe, private communication.
} 
directly map into our parameters $\theta$. 15 This implies that in our model the production cross section for $W^{* \pm}$ and $W^{* 3}$ (as well as their decay widths to light fermions), will be the same as that for the heavy vectors of Little Higgs models (see refs. [45, 46]). 16 On the other hand, the pattern of decays will be quite distinct in the two cases. In LH theories the couplings of $W^{*}$ to the various SM fermions are predicted to be either strictly universal (in product group models), or comparable in size (in simple group models). Partial compositeness, instead, predicts larger couplings to the third generation SM quarks, so that $t t, b b$ and $t b$ channels will have larger branching ratios. For example, in the case of $W^{* 3}$ one has (neglecting the SM fermion masses and the degree of compositeness of the SM leptons and light quarks):

$$
\begin{aligned}
& \Gamma\left(W^{* 3} \rightarrow q \bar{q}\right)=3 \Gamma\left(W^{* 3} \rightarrow l \bar{l}\right)=\frac{g_{2}^{2} M_{*}}{32 \pi} \tan ^{2} \theta_{2}, \\
& \Gamma\left(W^{* 3} \rightarrow t \bar{t}\right)=\Gamma\left(W^{* 3} \rightarrow b \bar{b}\right)=\frac{g_{2}^{2} M_{*}}{32 \pi}\left(\sin ^{2} \varphi_{t_{L}} \cot \theta_{2}-\cos ^{2} \varphi_{t_{L}} \tan \theta_{2}\right)^{2}, \\
& \Gamma\left(W^{* 3} \rightarrow Z h\right)=\Gamma\left(W^{* 3} \rightarrow W^{+} W^{-}\right)=\frac{g_{2}^{2} M_{*}}{192 \pi} \cot ^{2} \theta_{2}, \\
& \Gamma\left(W^{* 3} \rightarrow T \bar{t}\right)=\Gamma\left(W^{* 3} \rightarrow B \bar{b}\right)=\frac{g_{2}^{2} M_{*}}{32 \pi} \frac{\sin ^{2} \varphi_{t_{L}} \cos ^{2} \varphi_{t_{L}}}{\sin ^{2} \theta_{2} \cos ^{2} \theta_{2}}\left(1-\frac{1}{2} \frac{m_{*}^{2}}{M_{*}^{2}}-\frac{1}{2} \frac{m_{*}^{4}}{M_{*}^{4}}\right) \\
& \times\left(1-\frac{m_{*}^{2}}{M_{*}^{2}}\right), \\
& \Gamma\left(W^{* 3} \rightarrow \chi_{q} \bar{\chi}_{q}\right)=\frac{g_{2}^{2} M_{*}}{32 \pi}\left\{\left[\left(\cos ^{2} \varphi_{t_{L}} \cot \theta_{2}-\sin ^{2} \varphi_{t_{L}} \tan \theta_{2}\right)^{2}+\cot ^{2} \theta_{2}\right]\right. \\
& \left.\times\left(1-\frac{m_{*}^{2}}{M_{*}^{2}}\right)+6 \frac{m_{*}^{2}}{M_{*}^{2}}\left(\cos ^{2} \varphi_{t_{L}} \cot ^{2} \theta_{2}-\sin ^{2} \varphi_{t_{L}}\right)\right\} \\
& \times \sqrt{1-4 \frac{m_{*}^{2}}{M_{*}^{2}}} \\
& \Gamma\left(W^{* 3} \rightarrow \chi_{l} \bar{\chi}_{l}\right)=\frac{g_{2}^{2} M_{*}}{96 \pi} \cot ^{2} \theta_{2}\left(2+4 \frac{m_{*}^{2}}{M_{*}^{2}}\right) \sqrt{1-4 \frac{m_{*}^{2}}{M_{*}^{2}}}
\end{aligned}
$$

where $q(l)$ stands for any SM light quark (lepton) and $\chi_{q}=U_{i}, D_{i}, \chi_{l}=N_{i}, E_{i}$ for any flavor $i$. The first identity in the third equation follows from the Equivalence theorem: as pointed out above, final SM gauge bosons are almost completely longitudinally polarized.

\footnotetext{
${ }^{15}$ More precisely, the correspondence is $\theta_{L H}=\theta$ in the convention of Ref. [47, while $\theta_{L H}=\pi / 2-\theta$ in the notation of Refs. [45, 46, 48, 49.

16 Notice also that the electroweak precision data from LEP favor the region of parameter space of Little Higgs models where the second $S U(2) \times U(1)$ becomes strong, $\tan \theta_{L H} \ll 1$, and four-fermion contact interactions are thus suppressed [44, 50] (for more references on LH models and precision tests, see the review [35]). In other words, the region of parameter space where LH models pass the precision tests corresponds to that motivated by the partial compositeness paradigm.
} 
Naively, the LHC discovery reach in the channels $l \bar{l}, l \nu$ and $Z h, W W, W Z$ will be similar, though not identical, to that derived in Ref. [48] for the Littlest Higgs model [51]. In particular, the larger couplings to pairs of SM bosons and third-generation SM quarks imply a smaller branching fraction to the clean leptonic channels $l \bar{l}$. Despite the large branching ratio, $t t$ and $b b$ events will be certainly challenging to isolate over the huge SM background. On the other hand, detecting a large violation of flavor universality, as predicted in our model, would represent a first significant hint of partial compositeness. The $T t, B b, T b, B t$ channels should be easier to detect over the background, thanks to the richer final state. 17 If kinematically allowed, the decays to two heavy fermions would be instead extremely spectacular, especially those of $W^{* \pm}, W^{* 3}, \mathcal{B}^{*}$ to a pair of heavy leptons, which would translate to quite distinctive leptonic final states.

Ideally, one would like to measure several of these channels, in order to extract all the parameters and test the model. In the case of the $W^{* 3}$, for example, one can extract $\theta_{2}$ and $\varphi_{t_{L}}$ by counting the number of events in any two of the final states of Eq. (58); measuring a third channel then allows one to test the structure of the model. This ideal strategy must however confront with the difficulty of the actual experimental measurements. Quite likely, disentangling and measuring all the various parameters will be a hard task. A minimal strategy to verify the model after the discovery and discriminate it from other scenarios could be checking the relation:

$$
g_{H H \rho^{*}}^{2}=(g \cot \theta)^{2}=g_{H H \rho^{*} \rho^{*}},
$$

which is a direct consequence of the Higgs compositeness plus partial compositeness in the gauge sector. Similar tests have been also proposed for LH theories, see Refs. [45, 46]. The second equality in Eq. (59) is certainly harder to verify, due to the difficulty in measuring the coupling $g_{H H \rho^{*} \rho^{*}}$. The latter can be extracted from the associated production $q \bar{q} \rightarrow \rho^{*} \rightarrow$ $\rho^{*} V$, with $V=h, W_{L}, Z_{L}$.

Another unique feature of the partial compositeness scenario, as compared to other models like LH theories, is the existence of heavy excitations of the gluon, and the $\tilde{\rho}$ vectors. The former will have a larger cross section than the neutral weak excitations and will decay exclusively to $t t, b b$ and $T t, B b$ final states (and to two excited quarks if kinematically allowed). Very recently, Ref. [52] has made an in-depth study of the $t t$ channel and shown how to exploit a left-right polarization asymmetry, deriving from $\varphi_{t_{R}}>\varphi_{t_{L}}$, to efficiently extract the signal above SM background for gluon excitations of several TeV. As explained above, we expect the $T t, B b$ channels to also be favorable for detection, though a detailed study is needed. The heavy vectors $\tilde{\rho}$ are instead much more difficult to discover at the LHC because in their case there is no analog of the $A-\rho$ mixing, and their couplings to the light fermions will be extremely suppressed. The associated production $q \bar{q} \rightarrow \rho^{*} \rightarrow \tilde{\rho} V$, where $V=h, W_{L}, Z_{L}$, via a virtual or possibly real $\rho^{*}$, seems to be the most promising process, though a detailed study is again required.

\footnotetext{
${ }^{17}$ As we will discuss in detail below, heavy fermions mainly decay to a third-generation SM quark plus a longitudinally polarized gauge boson.
} 


\subsection{Heavy fermions production and decays}

If they are light enough, heavy excitations of the SM quarks will be pair produced at the LHC via QCD interactions:

$$
g g, q \bar{q} \rightarrow \chi \bar{\chi}
$$

and similarly for $\tilde{\chi}$. The cross section of these processes is completely determined as a function of the mass $m_{*}$ of the new particle, and falls off quickly as $m_{*}$ increases (see for example Refs. [46, 48]). An additional contribution comes from the exchange of a gluon excitation (similar diagrams with the exchange of a $W^{*}$ or a $\mathcal{B}^{*}$ are also possible, but smaller):

$$
q \bar{q} \rightarrow G^{*} \rightarrow \chi \bar{\chi}, \tilde{\chi} \overline{\tilde{\chi}}
$$

where $G^{*}$ can be real, if kinematically allowed. As discussed in the previous section, the electroweak precision tests directly constrain the mass of the top and bottom quark excitations, disfavoring values smaller than roughly $1.5 \mathrm{TeV}$. For such heavy masses, pair production of these states becomes small at the LHC. On the other hand, these same states couple strongly to their SM counterpart, and can be singly produced.

In the case of $\tilde{T}$ and $\tilde{B}$, an important role is played by $b W$ and $b Z$ fusion [53], where a bottom quark from one proton scatters off a longitudinal $W$ or $Z$ radiated by a quark from the other proton:

$$
\begin{aligned}
b_{L} W_{L} & \rightarrow \tilde{T}, & \lambda_{\tilde{T}} & =Y_{* U 33} \sin \varphi_{b_{L}} \cos \varphi_{t_{R}}=Y_{\text {top }} \cot \varphi_{t_{R}} \\
b_{L} Z_{L} & \rightarrow \tilde{B}, & \lambda_{\tilde{B}} & =Y_{* D 33} \sin \varphi_{b_{L}} \cos \varphi_{b_{R}}=Y_{\text {top }} \frac{Y_{* D 33}}{Y_{* U 33}} \frac{\cos \varphi_{b_{R}}}{\sin \varphi_{t_{R}}} .
\end{aligned}
$$

The same production mechanism has been discussed and studied in the context of Little Higgs models [46, 47, 48]. In order to make the comparison with the LH results easier, in Eq. (62) we have written the couplings relevant to each process, (they can be deduced from Eq. (32) by making use of the Equivalence theorem). 18 If $t_{R}$ is strongly coupled to the composite sector, $\sin \varphi_{t_{R}} \simeq 1$, then $\lambda_{\tilde{T}}$ is small and the single production of $\tilde{T}$ is suppressed. In fact, in the extreme limit in which $t_{R}$ is part of the composite sector, a heavy state $\tilde{T}$ is not required and we therefore removed it from our minimal model in Section 7 . The production rate of $\tilde{B}$ is instead expected to be sizable, since its coupling is large for $t_{R}$ composite and $b_{R}$ almost elementary: $\lambda_{\tilde{B}} \simeq Y_{\text {top }}\left(Y_{* D 33} / Y_{* U 33}\right) \approx 1$. In the case of $T$ and $B$, single production via $b W, b Z$ fusion will be extremely small if $b_{R}$ is almost elementary, and its couplings to the new states are weak. The analogous processes initiated by a $t_{R}$,

$$
t_{R} Z_{L} \rightarrow T, \quad t_{R} W_{L} \rightarrow B, \quad \lambda_{T}=\lambda_{B}=Y_{* U 33} \sin \varphi_{t_{R}} \cos \varphi_{t_{L}}=Y_{\text {top }} \cot \varphi_{t_{L}}
$$

might be large enough to be seen at the LHC, since the small top quark content of the proton can be compensated by the large coupling [9]: $\cot \varphi_{t_{L}} \gg 1$ for $t_{R}$ composite. A detailed analysis is however required. Single production of $B$ 's also proceeds via $g b_{L} \rightarrow B$,

\footnotetext{
${ }^{18}$ For comparison, the heavy $S U(2)_{L}$ singlet predicted by the Littlest Higgs model couples with strength $\lambda=Y_{\text {top }} \lambda_{1} / \lambda_{2}$, where $\lambda_{1} / \lambda_{2} \sim 1$.
} 
as the effect of the dimension-5 operator $g_{\mu \nu} \bar{B} \sigma^{\mu \nu} b$ generated by loops of top quarks. Finally, the associated production of a heavy $\chi=T, B$ or $\tilde{\chi}=\tilde{T}, \tilde{B}$ together with a SM top or bottom quark is also possible $(\psi=t, b)$ :

$$
q \bar{q} \rightarrow \rho^{*} \rightarrow \chi \psi, \tilde{\chi} \psi
$$

where $\rho^{*}=G^{*}, W^{*}, \mathcal{B}^{*}$ can be real. It is worth stressing that these processes, as well as all the other single production mechanisms, are strongly suppressed in the case of the excitations of SM light quarks, due to their small couplings to the composite sector. For them, only pair production will be viable. The same conclusion also applies to heavy leptonic resonances, with the difference that they can only be pair produced via the exchange of $S U(2)_{L} \times U(1)_{Y}$ carriers. 19

The decays of $T, B, \tilde{T}, \tilde{B}$ proceed through the same interaction vertices and couplings responsible for their production. The $S U(2)_{L}$ singlets $\tilde{T}$ and $\tilde{B}$ decay to a top or bottom quark plus a longitudinal SM boson or a Higgs, with branching ratios fixed by the Equivalence theorem:

$$
\begin{aligned}
\Gamma(\tilde{T} \rightarrow t h) & =\Gamma(\tilde{T} \rightarrow t Z)=\frac{1}{2} \Gamma(\tilde{T} \rightarrow b W)=\frac{\lambda_{\tilde{T}}^{2}}{32 \pi} \tilde{m}_{*}, \\
\Gamma(\tilde{B} \rightarrow b h) & =\Gamma(\tilde{B} \rightarrow b Z)=\frac{1}{2} \Gamma(\tilde{B} \rightarrow t W)=\frac{\lambda_{\tilde{B}}^{2}}{32 \pi} \tilde{m}_{*} .
\end{aligned}
$$

In addition to the above channels, the $\tilde{T}$ or $\tilde{B}$ can also decay to a $T, B$ if kinematically allowed. If the difference is mass between the two resonances is not too small, the eventual phase-space suppression can be compensated by the larger coupling involved: $Y_{* U, D}$. For example, if sufficiently heavier, a $\tilde{B}$ could decay to a $T(B)$ by emitting a $W_{L}\left(Z_{L}\right)$. This would represent a source of $T$ 's and $B$ 's, which might be otherwise difficult to produce, as we have seen. Furthermore, if $\tilde{T}, \tilde{B}$ undergo a decay chain instead of directly decaying to $\mathrm{SM}$ quarks, this leads to richer final states, which will be presumably easier to isolate over the SM background.

As already noticed in the literature [54, 48, 49, detecting the neutral-current decays of Eq. (65) would distinguish $\tilde{T}$ and $\tilde{B}$ from a fourth generation of quarks, though it would not be a smoking gun of partial compositeness. More peculiar to our scenario are the decays of $T$ and $B$ : in the motivated case of $b_{R}$ almost elementary, the only unsuppressed channels are:

$$
\Gamma(T \rightarrow t h)=\Gamma(T \rightarrow t Z)=\frac{\lambda_{T}^{2}}{32 \pi} m_{*}, \quad \Gamma(B \rightarrow t W)=\frac{\lambda_{B}^{2}}{16 \pi} m_{*},
$$

plus eventually the analogous decays to $\tilde{T}, \tilde{B}$, if kinematically allowed. Detecting only final states with top and no bottoms quarks from $T$ and $B$ would then be compelling evidence of partial compositeness and its mechanism for explaining the hierarchies in the SM Yukawas. On the other hand, directly checking the mechanism responsible for the cancellation of the

\footnotetext{
${ }^{19}$ Clearly, there is no analog of the processes (62) and 63 in the case of the heavy leptons, though they could be singly produced in the decays of $W^{*}$ and $\mathcal{B}^{*}$ if they had large couplings.
} 
quadratic divergence in the top loop will be challenging. A possible test in the case of $t_{R}$ composite consists in verifying the relation

$$
\lambda_{T}=Y_{\text {top }} \cot \varphi_{t_{L}}
$$

by using the value of $\varphi_{t_{L}}$ extracted, for example, from the decays of $\rho^{*}$. Determining $\lambda_{T}$, however, is not easy: a measurement from the total decay width is perhaps not possible, unless $\lambda_{T}$ is very large, due to the limited calorimeter resolution. One could use the production rate in the $t W, t Z$ fusion channels, if they are observed at LHC, although the theoretical error due to the uncertainties in the parton distribution functions will be probably large.

The scenario we have described so far assumes that flavor mixings in the composite sectors, induced by the off-diagonal entries of $Y_{*}$ in flavor space, are small. If this is the case, another evidence for partial compositeness could come from the production of the excitations of light quarks and leptons. Under the hypothesis of small flavor mixing in the composite sector, these heavy states are expected to be narrow resonances: they will decay to their SM counterpart plus a longitudinally weak boson or a Higgs, with much smaller couplings compared to those of Eqs. (65), (66). Most likely, flavor-changing decays to top and bottoms will also have sizable branching ratios, since the flavor suppression can be compensated by the larger coupling of the top and bottom to the composites. In the leptonic sector, pair production of the heavy leptons would give rise to spectacular signatures, by decaying to ultra-energetic SM leptons with essentially no SM background.

In the opposite, extreme limit of anarchic $Y_{*}$, flavor mixing effects among the composite fermions will be large. Single production of the heavy quark excitations will still proceed as discussed above, namely through a bottom or possibly a top quark from the proton, but the heavy $T, B, \tilde{T}, \tilde{B}$ produced in this way will easily mix with the excitations of the light quarks. These latter, on the other hand, will not decay anymore to light SM quarks, since it will be more convenient for them to change their flavor and decay to tops and bottoms. This scenario will then be distinguishable from the previous one for the absence of final states with light jets arising from the pair production of the first and second family quark excitations. Similar considerations in the case of pair production of heavy leptons show that these will mainly decay to taus, while muons and especially electrons in the final state will be rare.

We conclude by mentioning that the quark heavy excitations will produce also indirect effects, some of which might be spectacular, depending on the mass of the new particles. Important examples are a possible large modification to the Higgs production cross section via gluon fusion, and large shifts in the couplings of the top quark to the SM weak bosons.

\section{Acknowledgments}

We would like to thank Kaustubh Agashe, David E. Kaplan, Markus Luty, Alex Pomarol, Matthew Schwartz and Jay Wacker for useful discussions. We thank Stefania De Curtis for pointing out an error in the first version of the paper. This work was supported in part by the National Science Foundation grant NSF-PHY-0401513 and in part by the Johns Hopkins Theoretical Interdisciplinary Physics and Astrophysics Center. 


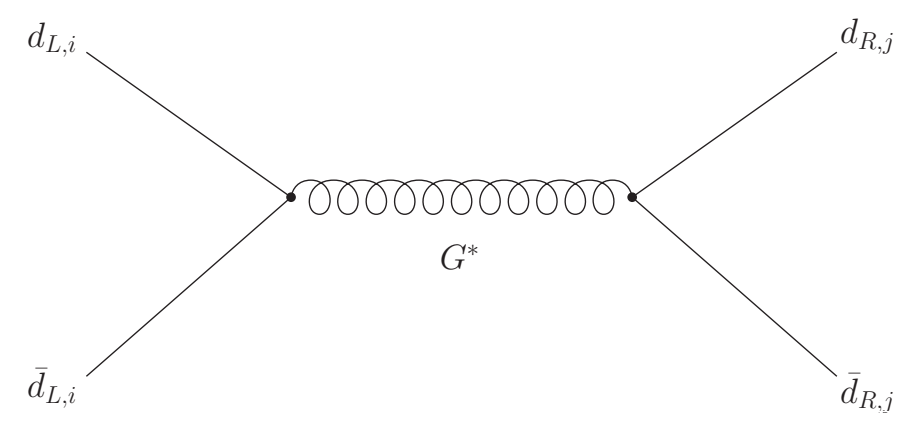

Figure 4: Tree-level exchange of an excited gluon, $G^{*}$, leading to the four-fermion LR operator of $E q .(68)$.

\section{A Generalized GIM mechanism for FCNCs}

Rather than re-discussing all the incarnations of partial compositeness in the phenomenology of low-energy flavor-changing processes, we will study just one class of examples to illustrate how the GIM mechanism of the SM is generalized in protecting against excessive flavorchanging neutral currents (FCNC's). The reader will thereby easily see how to compute other flavor-violating rare processes of interest from the Lagrangian of Eq. (31). We will focus here on four-fermion operators of the form

$$
\mathcal{L}_{F C N C}=A_{i j m n}\left(\bar{d}_{L i} \lambda_{a} \gamma_{\mu} d_{L j}\right)\left(\bar{d}_{R m} \lambda_{a} \gamma^{\mu} d_{R n}\right)
$$

arising by integrating out the exchange of an excited gluon, $G^{*}$, as in Fig. 4. The indices $i, j, m, n=1,2,3$ are generational, while $\lambda_{a}$ are the $S U(3)$ Gell-Mann matrices with the normalization of $\operatorname{Tr}\left(\lambda_{a} \lambda_{b}\right)=2 \delta_{a b}$.

Using the Lagrangian of Eq. (31), Fig. 4 yields (we consider only flavor-non-universal terms and work at leading order in $\theta_{3}$ )

$$
A_{i j m n} \simeq-\frac{g_{3}^{2} \cot ^{2} \theta_{3}}{4 M_{*}^{2}} \delta_{i j} \delta_{m n}\left(\sin ^{2} \varphi_{d_{L}}\right)_{i}\left(\sin ^{2} \varphi_{d_{R}}\right)_{m},
$$

but it must be borne in mind that this is in the gauge-eigenstate basis prior to EWSB. After EWSB, FCNCs will emerge upon converting to the mass-eigenstate basis as usual. Denoting by $S_{d_{L}}, S_{d_{R}}$ the $3 \times 3$ unitary transformations of the left- and right-handed down quarks that diagonalize the down-quark mass matrix, we have that in the mass-eigenstate basis

$$
A_{i j m n} \simeq-\frac{g_{3}^{2} \cot ^{2} \theta_{3}}{4 M_{*}^{2}}\left(S_{d_{L}}\right)_{k i}^{\dagger}\left(S_{d_{L}}\right)_{k j}\left(\sin ^{2} \varphi_{d_{L}}\right)_{k}\left(\sin ^{2} \varphi_{d_{R}}\right)_{l}\left(S_{d_{R}}\right)_{l m}^{\dagger}\left(S_{d_{R}}\right)_{l n} .
$$

We can then see how the generalized flavor protection works for the lighter fermions. Flavorchanging neutral currents arise via $S_{d_{L}}, S_{d_{R}}$, but also depends on the partial compositeness of the fermions, $\left(\sin \varphi_{d_{L}}\right)_{i},\left(\sin \varphi_{d_{R}}\right)_{i} \neq 0$. The lighter fermions are mostly elementary, with weak couplings to the Higgs, $\left|\left(\varphi_{d_{L, R}}\right)_{i}\right| \ll 1$. Thus FCNCs are suppressed the lighter the fermions involved. 


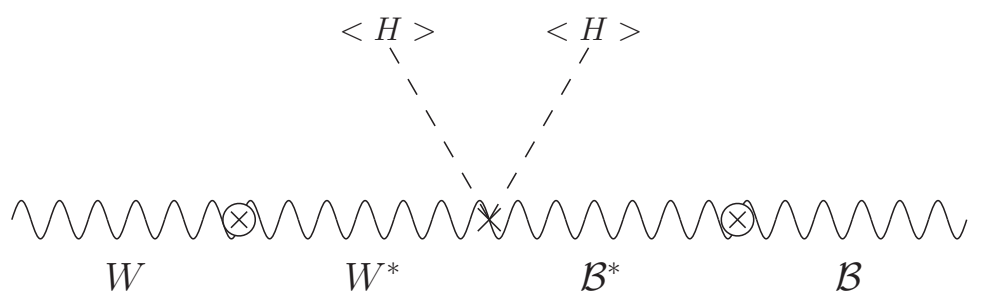

Figure 5: Tree-level contribution to $S$.

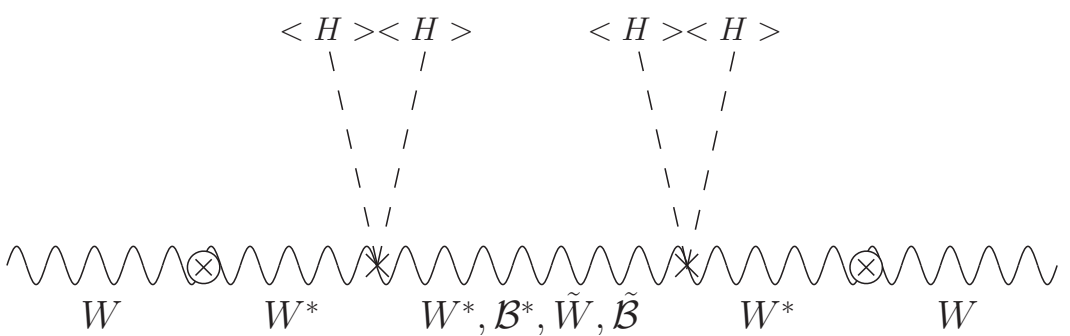

Figure 6: Tree-level contribution to $T$. The $S U(2)_{L} \times S U(2)_{R}$ invariance of the composite gauge sector ensures that this contribution exactly vanishes.

\section{B Oblique corrections}

The leading oblique corrections can be parametrized by the Peskin-Takeuchi $S$ and $T$ parameters [42]. One useful aspect of these variables is that they are defined directly in terms of vacuum polarizations, rather than in terms of corrections to the physical SM gauge couplings. This is done by adopting an oblique field basis, which is precisely the basis in which all the universal corrections to the gauge couplings of the SM light fermions can be encoded in the electroweak vacuum polarizations. Quite interestingly, the oblique basis of Peskin and Takeuchi essentially coincides with the elementary/composite basis, apart from highly-suppressed corrections due to the small composite component of the physical light fermions. This means that the new-physics contributions to these variables can be obtained more readily by using the Lagrangian before mass-diagonalization.

The leading new-physics contribution to the $S$ parameter is given by the mixing diagram of Fig. [5, and yields

$$
S=4 \pi v^{2}\left(\frac{1}{M_{* 1}^{2}}+\frac{1}{M_{* 2}^{2}}\right) .
$$

We can already foresee one central feature of the spectrum relevant for the LHC: the composite vector mesons must be quite heavy, $M_{*} \gtrsim 2.3 \mathrm{TeV}$ in order to keep $S \lesssim 0.3$, as required at the $99 \%$ CL by the precision data (see for example [44).

The tree-level contribution to the $T$ parameter is given by the diagram of Fig. [6, but the custodial symmetry of the composite sector implied by the $\left[S U(2)_{L} \otimes S U(2)_{R}\right]^{\text {comp }}$ global 


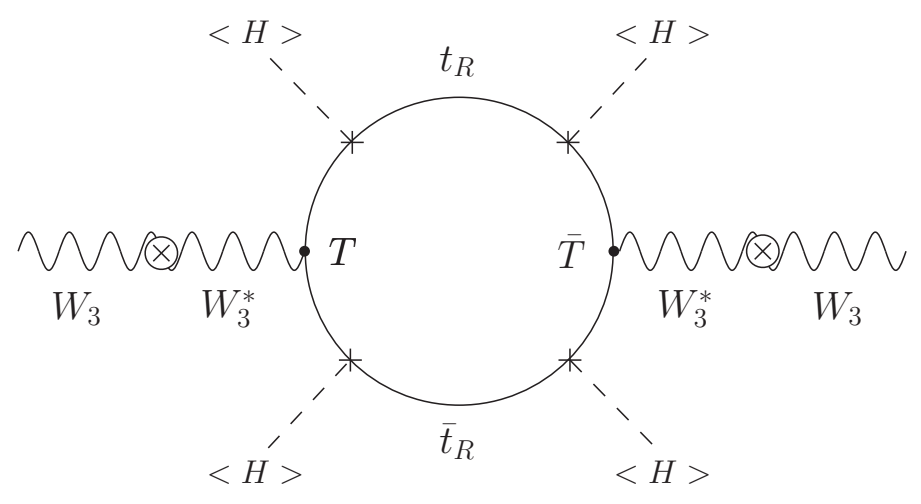

Figure 7: One-loop dominant contribution to $T$.

invariance ensures that there is a complete cancellation:

$$
T=0 \quad \text { at tree level } .
$$

In fact, the suppression of $T$ at the tree level by a custodial isospin symmetry is the motivation for extending the composite gauge group. The custodial symmetry of the composite gauge sector is however spoiled at the loop level, since all composite fermions are singlets of $\left[S U(2)_{R}\right]^{c o m p}$ and their Yukawa couplings to the Higgs break explicitly the custodial symmetry. The dominant one-loop contribution to the $T$ parameter is given by the diagram of Fig. 7, which gives

$$
T=\frac{2}{3} T_{S M}^{t o p}\left(\frac{Y_{* U 33}^{2}}{Y_{\text {top }}}\right)^{2}\left(\frac{v}{m_{33}^{Q}}\right)^{2},
$$

where

$$
T_{S M}^{t o p}=\frac{3}{16 \pi \sin ^{2} \theta_{w} \cos ^{2} \theta_{w}} \frac{m_{t}^{2}}{m_{Z}^{2}} \simeq 1.2 .
$$

\section{Non-universal corrections: $Z \rightarrow b_{L} \bar{b}_{L}$}

Non-universal effects are most important for the quarks of the third generation, since these are the fermions with the largest mixing with the composite sector. In particular, the strongest constraint comes from the $Z b_{L} \bar{b}_{L}$ coupling, which has been quite precisely measured by LEP and SLD experiments. We follow the usual convention and define the left-handed coupling $g_{L b}$ so that its SM value at tree level is $g_{L b}=T^{3 L}-Q \sin ^{2} \theta_{w}$. After EWSB $g_{L b}$ receives a correction due to the mixing of $b_{L}$ with the heavy electroweak singlet $\tilde{B}$. This shift is of the form

$$
\delta g_{L b}=\sin ^{2} \xi\left(T^{3 L}(\tilde{B})-T^{3 L}\left(b_{L}\right)\right),
$$




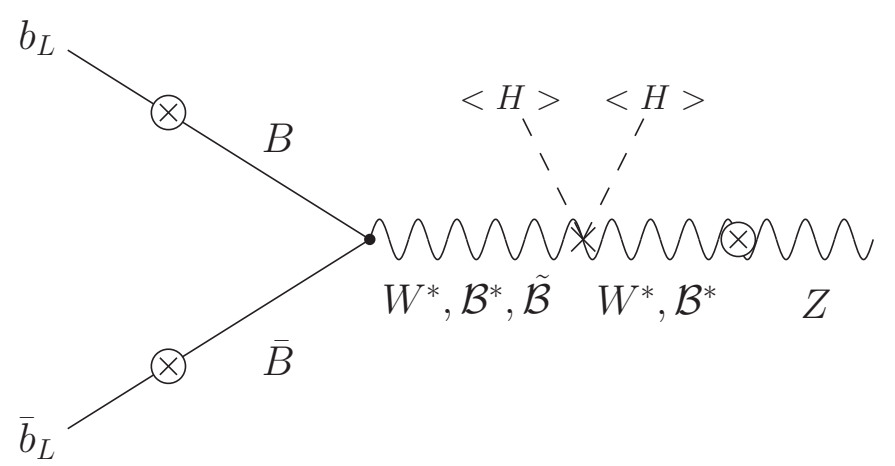

Figure 8: Tree-level contributions to $\delta g_{L}$ from the exchange of gauge excitations, in the insertion approximation. A circled cross denotes a mass mixing.

where $T^{3 L}(\tilde{B})=0$, and the mixing angle $\xi$ between $b_{L}$ and $\tilde{B}_{L}$ can be extracted from the mass matrix (44). At leading order in $\left(v \sin \varphi_{b_{L}} Y_{* D 33} / \tilde{m}_{*}^{B}\right)$ we obtain:

$$
\xi \simeq \frac{Y_{* D 33}}{\tilde{m}_{*}^{B}} \frac{v}{\sqrt{2}} \sin \varphi_{b_{L}} \cos \varphi_{b_{R}} .
$$

Another correction to $g_{L b}$ comes from the mixing of the $Z$ with $W^{* 3}, B^{*}$ and $\mathcal{B}$ after EWSB. This shift is most conveniently computed adopting the elementary/composite basis, and working for simplicity at leading order in the elementary/composite mass insertions $\left(\Delta_{b_{L}} / m^{Q}\right)$ and $\left(g_{e l} / g_{*}\right)$. The leading contribution is given by the diagram of Fig. 8, which, added to the fermionic one, leads to (for $t_{R}$ fully composite)

$$
\delta g_{L b} \simeq \frac{1}{2}\left(\frac{Y_{* D 33}}{Y_{* U 33}}\right)^{2}\left(\frac{m_{t}}{\tilde{m}_{*}^{B}}\right)^{2}+\frac{1}{4}\left(\frac{m_{t}}{M_{* 2}}\right)^{2}\left(\frac{g_{* 2}}{Y_{* U 33}}\right)^{2} .
$$

\section{Explicit gauge-invariance breaking and UV cutoff}

While our model's defining Lagrangian has all explicit couplings and mass parameters with non-negative mass dimensions (naively a check of renormalizability), the model is still nonrenormalizable because $M_{*}, Y_{*}$ and the mixing masses explicitly break the composite and elementary gauge symmetries, preserving only the subgroup of SM gauge symmetries. Therefore, the quantum theory becomes strongly coupled in the UV and there is a maximal energy, $\Lambda_{U V}$ before losing perturbative control. In this appendix we estimate this effective UV cutoff on the consistency of our model and show that it is well above the energies we wish to study. Of course, cutoff physics can also appear virtually in precision effects, but these will be suppressed relative to $M_{*}$-scale contributions by $\mathcal{O}\left(M_{*}^{2} / \Lambda_{U V}^{2}\right)$.

The strongest couplings arise from the composite sector, so for the present exercise we focus on this sector exclusively, in particular the breaking of composite gauge invariance due to $M_{*}, Y_{*}$. We can always imagine that the composite Lagrangian is just the unitary 
gauge representation of a new Higgs mechanism (distinct from the electroweak Higgs mechanism of course). One can "go back" to the un-gauge-fixed theory by performing a general gauge transformation, of general form $\exp \left(i \Pi(x) . \mathcal{T} / F_{*}\right)$, on the composite Lagrangian, and interpreting the spacetime-dependent parameters $\Pi(x)$ as the eaten Goldstone bosons. For example,

$$
\frac{M_{*}^{2}}{2} \rho_{\mu}^{2} \rightarrow \frac{M_{*}^{2}}{g_{*}^{2}}\left|D_{\mu} e^{i \Pi \cdot \mathcal{T} / F_{*}}\right|^{2},
$$

the leading term in a now explicitly non-renormalizable (gauged) chiral Lagrangian. The Goldstone bosons, $\Pi(x)$, are canonically normalized if we take their "decay constant", $F_{*}$, to satisfy

$$
M_{*}=\frac{g_{*} F_{*}}{2} .
$$

We can now use naive dimensional analysis [55] to estimate when the non-renormalizable interactions become non-perturbatively strong. The standard result for the non-renormalizable chiral Lagrangian is

$$
\Lambda_{U V} \sim 4 \pi F_{*}=\frac{8 \pi M_{*}}{g_{*}}, \quad \Lambda_{U V} \gg M_{*} \text { for } g_{*} \ll 4 \pi .
$$

Similarly, the un-gauge-fixed composite Yukawa coupling is of the general form

$$
Y_{*} \bar{\chi} H \tilde{\chi} \rightarrow Y_{*} \bar{\chi} H e^{i \Pi \cdot \mathcal{T} / F_{*}} \tilde{\chi}
$$

Such a non-renormalizable coupling renormalizes itself (at two-loop order). For the divergent loop corrections to be weaker than the tree coupling, the cutoff must satisfy

$$
\frac{Y_{*}^{3} \Lambda_{U V}^{2}}{\left(16 \pi^{2}\right)^{2} F_{*}^{2}}<Y_{*}
$$

The implied cutoff, $\Lambda_{U V}<16 \pi^{2} F_{*} / Y_{*}$, is subdominant to Eq. (80).

We conclude that our model is a weakly coupled non-renormalizable effective field theory up to an energy scale given roughly by Eq. (80), which is over $10 \mathrm{TeV}$ for the parameter range of interest.

\section{E Randall-Sundrum graviton and radion excitations}

The model we have presented in this paper is the minimal one realizing the partial compositeness of SM fields. The original Randall-Sundrum warped compactification model [1] focussed instead on the presence of observable graviton excitations at the TeV scale, needed to realize partial compositeness of the ordinary massless graviton. We have not included the lightest graviton excitation because the massless graviton is itself too weakly coupled to be relevant at particle colliders and because the more strongly coupled graviton excitation is SM-neutral and less important in the face of so many SM-charged excitations, as in warped compactifications with "bulk" SM fields. The original Randall-Sundrum model also 
possessed a light SM-neutral "radion" scalar, although its presence is dependent on details of stabilization of the size of the warped compactification, so again we have not included it thus far. Moreover, it is not needed to realize partial compositeness of just the SM. However, both the graviton and radion excitations have interesting properties and may be visible at colliders, and therefore deserve further consideration. We briefly indicate how they are to be included in the present context.

From the compositeness viewpoint, the graviton and radion excitations are pure spin-2 and spin-0 composites respectively. (There is entirely negligible mixing with the massless elementary graviton.) They are included in our model by modifying the composite sector and mixing terms,

$$
\begin{aligned}
& \mathcal{L}_{\text {composite }}+\mathcal{L}_{\text {mixing }} \rightarrow \sqrt{-G}\left\{\tilde{\mathcal{L}}_{\text {composite }}+\tilde{\mathcal{L}}_{\text {mixing }}+2 M_{\text {Pl* }}^{2} R\right\} \\
& -\frac{\mathcal{M}_{*}^{2}}{4}\left(1+\frac{\phi}{F}\right)^{4}\left(H_{\mu \nu} H^{\mu \nu}-H_{\mu}^{\mu} H_{\nu}^{\nu}\right)+\left(\frac{1}{2}+\frac{12 M_{P l *}^{2}}{F^{2}}\right)\left(\partial_{\mu} \phi\right)^{2}-V_{G W}(\phi) .
\end{aligned}
$$

The first line is generally coordinate invariant with respect to a "metric" field

$$
G_{\mu \nu} \equiv\left(\eta_{\mu \nu}+\frac{H_{\mu \nu}}{M_{P l *}}\right)\left(1+\frac{\phi}{F}\right)^{2},
$$

which houses both the symmetric Lorentz-tensor graviton field, $H_{\mu \nu}$, and the radion scalar, $\phi$. The $\tilde{\mathcal{L}}$ terms are minimally coupled to the metric, while the $R$ term is the Ricci scalar term made from $G_{\mu \nu}$, acting as a kinetic term for the graviton. In this field normalization, the graviton "Planck mass" parameter of several TeV controls the coupling of the graviton to itself and other fields. The second line however breaks the general coordinate invariance, by the graviton "Pauli-Fierz" mass term [56], with mass $\mathcal{M}_{*}>\mathrm{TeV}$, a radion kinetic term (not coupled to the graviton), and a radion potential. The radion kinetic term appears peculiarly normalized because it also gets a contribution from the $R$ term on the first line. The deconstructed form of the light Randall-Sundrum graviton excitation was proposed in Ref. [57]. From the compositeness viewpoint it relates [58] to the strong interactions approach of Tensor Meson Dominance [59].

If we turn off all the elementary sector fields as well as $V_{G W}$, and focus on the composite sector alone, the radion field is coupled consistently with it being the Goldstone boson of spontaneous conformal-symmetry breaking in the composite sector. This is the composite dynamics dual role of the Randall-Sundrum radion [21, 60]. That is, the physical picture is that the composite sector is strongly coupled but conformally-invariant until this conformalsymmetry breaking at a scale $F$, below which the composites are generally massive, but coupled to the massless Goldstone boson, $\phi$. The radion potential,

$$
V_{G W}=\frac{1}{2} m_{\phi}^{2} \phi^{2}+\mu_{\phi} \phi^{3}+\lambda_{\phi} \phi^{4}
$$

represents a weak explicit breaking of the Goldstone symmetry as exemplified in the RandallSundrum context by the well-known Goldberger-Wise (GW) stabilization mechanism [61]. 
There is a standard subtlety in coupling the (composite) fermions to the graviton excitation, in that one cannot work in terms of a metric field, but rather must use a vierbein,

$$
E_{\mu}^{a}=\delta_{\mu}^{a}+\tilde{H}_{\mu}^{a},
$$

which is related to the metric field by

$$
G_{\mu \nu} \equiv \eta_{a b} E_{\mu}^{a} E_{\nu}^{b}
$$

In standard fashion, both metric and vierbein house the same physical graviton field, plus extra off-shell degrees of freedom, but the (more cumbersome) vierbein is useful for covariantizing fermion kinetic terms.

With the above modification of the composite and mixing Lagrangians, the reader can easily derive the couplings of the SM and the SM-charged excitations to the radion and graviton. Note that some of the most important couplings of the radion to the SM fields will appear at the loop level. For example, it is straightforward to check that after mass diagonalization the radion-gluon-gluon coupling vanishes classically, but it is generated via quantum loops of heavy colored excitations.

\section{References}

[1] L. Randall and R. Sundrum, Phys. Rev. Lett. 83, 3370 (1999) arXiv:hep-ph/9905221.

[2] H. Davoudiasl, J. L. Hewett and T. G. Rizzo, Phys. Lett. B 473, 43 (2000) arXiv:hep-ph/9911262; S. Chang et al., Phys. Rev. D 62, 084025 (2000) arXiv:hep-ph/9912498.

[3] Y. Grossman and M. Neubert, Phys. Lett. B 474, 361 (2000) arXiv:hep-ph/9912408.

[4] T. Gherghetta and A. Pomarol, Nucl. Phys. B 586, 141 (2000) arXiv:hep-ph/0003129].

[5] S. J. Huber and Q. Shafi, Phys. Lett. B 498, 256 (2001) arXiv:hep-ph/0010195], S. J. Huber, Nucl. Phys. B 666, 269 (2003) arXiv:hep-ph/0303183].

[6] G. Burdman, Phys. Rev. D 66, 076003 (2002) [arXiv:hep-ph/0205329]; Phys. Lett. B 590, 86 (2004) arXiv:hep-ph/0310144]; K. Agashe, G. Perez and A. Soni, Phys. Rev. Lett. 93, 201804 (2004) [arXiv:hep-ph/0406101]; Phys. Rev. D 71, 016002 (2005) arXiv:hep-ph/0408134]; arXiv:hep-ph/0606293; K. Agashe, M. Papucci, G. Perez and D. Pirjol, arXiv:hep-ph/0509117.

[7] S. J. Huber and Q. Shafi, Phys. Rev. D 63, 045010 (2001) arXiv:hep-ph/0005286]; S. J. Huber and C. -A. Lee and Q. Shafi, Phys. Lett. B 531, 112 (2002) arXiv:hep-ph/0111465]; C. Csaki, J. Erlich and J. Terning, Phys. Rev. D 66, 064021 (2002) |arXiv:hep-ph/0203034]; J. L. Hewett, F. J. Petriello and T. G. Rizzo, JHEP 0209, 030 (2002) |arXiv:hep-ph/0203091]. 
[8] K. Agashe, A. Delgado, M. J. May and R. Sundrum, JHEP 0308, 050 (2003) arXiv:hep-ph/0308036.

[9] K. Agashe, R. Contino and A. Pomarol, Nucl. Phys. B 719, 165 (2005) arXiv:hep-ph/0412089].

[10] K. Agashe and R. Contino, Nucl. Phys. B 742, 59 (2006) arXiv:hep-ph/0510164.

[11] K. Agashe, R. Contino, L. D. Rold and A. Pomarol, Phys. Lett. B 641, 62 (2006) arXiv:hep-ph/0605341.

[12] A. Pomarol, Phys Rev. Lett. 85, 4004 (2000) arXiv:hep-ph/0005293]; L. Randall and M. Schwartz, JHEP 0111, 003 (2001) [arXiv:hep-th/0108114 and Phys. Rev. Lett. 88, 081801 (2002) |arXiv:hep-th/0108115|; W. Goldberger and I. Rothstein, Phys Rev. Lett. 89, 131601 (2002) [arXiv:hep-th/0204160]; Phys Rev. D 68, 125011 (2003) arXiv:hep-th/0208060]; K. Agashe, A. Delgado and R. Sundrum, Nucl Phys. B 643, 172 (2002) |arXiv:hep-ph/0206099]; R. Contino, P. Creminelli and E. Trincherini, JHEP 0210, 029 (2002) [arXiv:hep-th/0208002]; K. Choi and I-W. Kim, Phys Rev. D 67, 045005 (2003) arXiv:hep-th/0208071|; W. D. Goldberger, Y. Nomura and D. R. Smith, Phys Rev. D 67, 075021 (2003) |arXiv:hep-ph/0209158]; K. Agashe, A. Delgado and R. Sundrum, Annals Phys. 304, 145 (2003) |arXiv:hep-ph/0212028].

[13] K. Agashe, R. Contino and R. Sundrum, Phys. Rev. Lett. 95, 171804 (2005) arXiv:hep-ph/0502222.

[14] K. Agashe and G. Servant, Phys. Rev. Lett. 93, 231805 (2004) arXiv:hep-ph/0403143; JCAP 0502, 002 (2005) arXiv:hep-ph/0411254.

[15] I. Antoniadis, Phys. Lett. B 246, 377 (1990); J. D. Lykken, Phys. Rev. D 54, 3693 (1996) arXiv:hep-th/9603133.

[16] N. Arkani-Hamed, S. Dimopoulos and G. R. Dvali, Phys. Lett. B 429, 263 (1998) arXiv:hep-ph/9803315]; I. Antoniadis, N. Arkani-Hamed and S. Dimopoulos, Phys. Lett. B 436, 257 (1998) arXiv:hep-ph/9804398.

[17] N. Arkani-Hamed and M. Schmaltz, Phys. Rev. D 61, 033005 (2000) arXiv:hep-ph/9903417]; E. A. Mirabelli and M. Schmaltz, Phys. Rev. D 61, 113011 (2000) |arXiv:hep-ph/9912265].

[18] Y. Hosotani, Phys. Lett. B 129, 193 (1983); Annals. Phys. 190, 233 (1989).

[19] J. Maldacena, Adv. Theor. Math. Phys. 2, 231 (1998) arXiv:hep-th/9711200; S. Guber, I. Klebanov and A. Polyakov, Phys. Lett. B 428, 105 (1998) [arXiv:hep-th/9802109]; E. Witten, Adv. Theor. Math. Phys. 2, 253 (1998) |arXiv:hep-th/9802150|; for a review see O. Aharony, S. Gubser, J. Maldacena, H. Oguri and Y. Oz, Phys. Pept. 323, 183 (2000) arXiv:hep-th/9905111. 
[20] H. Verlinde, Nucl. Phys. B 580, 264 (2000) arXiv:hep-th/9906182; J. Maldacena, unpublished remarks; E. Witten, ITP Santa Barbara conference 'New Dimensions in Field Theory and String Theory', http://www.itp.ucsb.edu/online/susy c99/discussion/ ; S. Gubser, Phys. Rev. D 63, 084017 (2001) arXiv:hep-th/9912001; E. Verlinde and H. Verlinde, JHEP 0005, 034 (2000) arXiv:hep-th/9912018].

[21] N. Arkani-Hamed, M. Porrati and L. Randall, JHEP 0108, 017 (2001) arXiv:hep-th/0012148; R. Rattazzi and A. Zaffaroni, JHEP 0104, 021 (2001) arXiv:hep-th/0012248.

[22] M. Perez-Victoria, JHEP 0105, 064 (2001) arXiv:hep-th/0105048].

[23] S. Weinberg, Phys. Rev. D13, 974 (1976); L. Susskind, Phys. Rev. D 20, 2619 (1979).

[24] B. Holdom, Phys. Lett. B 150, 301 (1985); T. Appelquist, D. Karabali and L. C. R. Wijewardhana, Phys. Rev. D 35, 2605 (1987); M. Bando, T. Morozumi, H. So and K. Yamawaki, Phys. Rev. Lett. 59, 389 (1987); V. A. Miransky, Nuovo Cimento A 90, 149 (1985); T. Appelquist and L. C. R. Wijewardhana, Phys. Rev. D 36, 568 (1987).

[25] C. Csaki, C. Grojean, L. Pilo and J. Terning, Phys. Rev. Lett. 92, 101802 (2004) arXiv:hep-ph/0308038.

[26] H. Georgi and D. B. Kaplan, Phys. Lett. B 136, 183 (1984) and Phys. Lett. B 145, 216 (1984); S. Dimopoulos, H. Georgi and D. B. Kaplan, Phys. Lett. B136, 187 (1984); P. Galison, H. Georgi and D. B. Kaplan, Phys. Lett. B 143, 152 (1984); M. J. Dugan, H. Georgi and D. B. Kaplan, Nucl. Phys. B 254, 299 (1985).

[27] R. Contino, Y. Nomura and A. Pomarol, Nucl. Phys. B 671, 148 (2003) arXiv:hep-ph/0306259.

[28] Y. Nambu, report EFI 88-39 (July 1988), published in the preceedings of the Kazimierz 1988 Conference on New Theories in physics, ed. T. Eguchi and K. Nishijima; in the preceedings of the 1988 International workshop on New Trends in Strong Coupling Gauge Theories, Nagoya, Japan, ed. Bando, Muta, and Yamawaki (World Scientific, 1989); report EFI-89-08 (1989); V. A. Miransky, M. Tanabashi and K. Yamawaki, Mod. Phys. Lett. A 4, 1043(1989); Phys. Lett. B 221, 177 (1989); W. J. Marciano, Phys. Lett. 62, 2793 (1989).

[29] W. A. Bardeen, C. T. Hill and M. Linder, Phys. Rev. D 41, 1647 (1990).

[30] C. T. Hill, Phys. Lett. B 266, 419 (1991).

[31] D. B. Kaplan, Nucl. Phys. B 365, 259 (1991).

[32] N. Arkani-Hamed, A. G. Cohen and H. Georgi, Phys. Rev. Lett. 86, 4757 (2001) arXiv:hep-th/0104005]; C. T. Hill, S. Pokorski and J. Wang, Phys. Rev. D 64, 105005 (2001) |arXiv:hep-th/0104035. 
[33] H. C. Cheng, C. T. Hill and J. Wang, Phys. Rev. D 64, 095003 (2001) arXiv:hep-ph/0105323; ; H. Abe, T. Kobayashi, N. Maru and K. Yoshioka, arXiv:hep-ph/0205344; A. Falkowski and H. D. Kim, JHEP 0208, 052 (2002) arXiv:hep-ph/0208058; L. Randall, Y. Shadmi and N. Weiner, JHEP 0301, 055 (2003) [arXiv:hep-th/0208120]; A. Katz and Y. Shadmi, JHEP 0411, 060 (2004) [arXiv:hep-th/0409223]; J. de Blas, A. Falkowski, M. Perez-Victoria and S. Pokorski, JHEP 0608, 061 (2006) [arXiv:hep-th/0605150].

[34] N. Arkani-Hamed, A. G. Cohen and H. Georgi, Phys. Lett. B 513, 232 (2001) arXiv:hep-ph/0105239].

[35] M. Schmaltz and D. Tucker-Smith, Ann. Rev. Nucl. Part. Sci. 55, 229 (2005) arXiv:hep-ph/0502182].

[36] M. Bando, T. Kugo, S. Uehara, K.Yamawaki and T. Yanagida, Phys. Rev. Lett. 54, 1215 (1985).

[37] R. Casalbuoni, S. De Curtis, D. Dominci and R. Gatto, Phys. Lett. B 155, 95 (1985); Nucl. Phys. B 282, 235 (1987).

[38] K. D. Lane, Phys. Rev. D 60, 075007 (1999) arXiv:hep-ph/9903369]; K. Lane and S. Mrenna, Phys. Rev. D 67, 115011 (2003) [arXiv:hep-ph/0210299].

[39] R. S. Chivukula, B. Coleppa, S. D. Chiara, E. H. Simmons, H. He, M. Kurachi and M. Tanabashi, Phys. Rev. D 74, 075011 (2006) arXiv:hep-ph/0607124.

[40] H.-C. Cheng, J. Thaler and L.-T. Wang, JHEP 0609, 003 (2006) arXiv:hep-ph/0607205].

[41] R. Barbieri and A. Strumia, arXiv:hep-ph/0007265.

[42] M. E. Peskin and T. Takeuchi, Phys. Rev. Lett. 65, 964 (1990); Phys. Rev. D 46, 381 (1992).

[43] The LEP Electroweak Working Group, CERN-PH-EP/2005-051 and hep-ex/0511027 (November 2005) updated for 2006 summer conferences; http://lepewwg. web. cern. ch/LEPEWWG.

[44] R. Barbieri, A. Pomarol, R. Rattazzi and A. Strumia, Nucl. Phys. B 703, 127 (2004) arXiv:hep-ph/0405040].

[45] G. Burdman, M. Perelstein and A. Pierce, Phys. Rev. Lett. 90, 241802 (2003) [Erratumibid. 92, 049903 (2004)] arXiv:hep-ph/0212228.

[46] T. Han, H. E. Logan, B. McElrath and L. T. Wang, Phys. Rev. D 67, 095004 (2003) arXiv:hep-ph/0301040]. 
[47] M. Perelstein, M. E. Peskin and A. Pierce, Phys. Rev. D 69, 075002 (2004) arXiv:hep-ph/0310039.

[48] G. Azuelos et al., Eur. Phys. J. C 39S2, 13 (2005) arXiv:hep-ph/0402037].

[49] T. Han, H. E. Logan and L. T. Wang, JHEP 0601, 099 (2006) arXiv:hep-ph/0506313.

[50] Z. Han and W. Skiba, Phys. Rev. D 72, 035005 (2005) arXiv:hep-ph/0506206];

G. Marandella, C. Schappacher and A. Strumia, Phys. Rev. D 72, 035014 (2005) arXiv:hep-ph/0502096.

[51] N. Arkani-Hamed, A. G. Cohen, E. Katz and A. E. Nelson, JHEP 0207, 034 (2002) arXiv:hep-ph/0206021].

[52] K. Agashe, A. Belyaev, T. Krupovnickas, G. Perez, J. Virzi, arXiv:hep-ph/0612015.

[53] S. S. D. Willenbrock and D. A. Dicus, Phys. Rev. D 34, 155 (1986).

[54] F. del Aguila, G. L. Kane and M. Quiros, Phys. Rev. Lett. 63, 942 (1989).

[55] A. Manohar and H. Georgi, Nucl. Phys. B 234, 189 (1984) arXiv:hep-ph/0510164;

H. Georgi and L. Randall, Nucl. Phys. B 276, 241 (1996).

[56] M. Fierz and W. Pauli, Proc. Roy. Soc. Lond. A 173, 211 (1939).

[57] N. Arkani-Hamed, H. Georgi, M. Schwartz, Ann. Phys. 305, 96 (2003) arXiv:hep-th/0210184.

[58] E. Katz, A. Lewandowski, M. Schwartz, Phys. Rev. D 74, 086004 (2006) arXiv:hep-ph/0510388.

[59] M. Suzuki, Phys. Rev. D 47, 1043 (1993).

[60] R. Sundrum, arXiv:hep-th/0312212.

[61] W. Goldberger, M. B. Wise, Phys. Rev. D 60, 107505 (1999) arXiv:hep-ph/9907218. 